Pacific

Journal of

Mathematics

FUNDAMENTAL SOLUTIONS OF INVARIANT DIFFERENTIAL OPERATORS ON A SEMISIMPLE LIE GROUP

Guillermo Ames

Volume 196 No. 1

November 2000 


\title{
FUNDAMENTAL SOLUTIONS OF INVARIANT DIFFERENTIAL OPERATORS ON A SEMISIMPLE LIE GROUP
}

\author{
Guillermo Ames
}

Let $G$ be a connected semisimple Lie group of real rank one. We denote by $\mathcal{U}(\mathfrak{g})^{K}$ the algebra of left invariant differential operators on $G$ right invariant by $K$, and let $\mathcal{Z}\left(\mathcal{U}(\mathfrak{g})^{K}\right)$ be its center.

In this paper we give a sufficient condition for a differential operator $P \in \mathcal{Z}\left(\mathcal{U}(\mathfrak{g})^{K}\right)$ to have a fundamental solution on $G$. We verify that this condition implies $P C^{\infty}(G)=C^{\infty}(G)$. If $G$ has a compact Cartan subgroup, we also give a sufficient condition for a differential operator $P \in \mathcal{Z}\left(\mathcal{U}(\mathfrak{g})^{K}\right)$ to have a parametrix on $G$. Finally we prove a necessary condition for the existence of parametrix of $P \in \mathcal{Z}\left(\mathcal{U}(\mathfrak{g})^{K}\right)$ for a connected semisimple Lie group.

\section{Introduction.}

Let $G$ be a connected semisimple Lie group. The algebra of left invariant differential operators on $G$ identifies canonically with the universal algebra $\mathcal{U}(\mathfrak{g})$. The operators of the center $\mathcal{Z}(\mathfrak{g})$ are the bi-invariant differential operators on $G$, i.e., left and right invariant. More generally, we consider the algebra $\mathcal{U}(\mathfrak{g})^{K}$ of right $K$-invariant differential operators of $\mathcal{U}(\mathfrak{g})$, where $K$ is a maximally compact subgroup of $G$, and $\mathcal{Z}\left(\mathcal{U}(\mathfrak{g})^{K}\right)$ will denote its center.

We denote by $\mathcal{D}(G)$ the space of $C^{\infty}$ functions with compact support. The dual $\mathcal{D}^{\prime}(G)$ of continuous linear functionals in $\mathcal{D}(G)$ is the space of distributions of $G$.

An operator $P$ in $\mathcal{U}(\mathfrak{g})$ acts on $\mathcal{D}^{\prime}(G)$ in the following way:

$$
P T(f)=T\left(P^{t} f\right),
$$

where $P^{t} \in \mathcal{U}(\mathfrak{g})$ is such that, if $d x$ is a Haar measure on $G$,

$$
\int_{G} P f(x) g(x) d x=\int_{G} f(x) P^{t} g(x) d x .
$$

If $X \in \mathfrak{g}, X^{t}=-X$, so the $P \mapsto P^{t}$ is the anti automorphism of $\mathcal{U}(\mathfrak{g})$ extending $-I d$ of $\mathfrak{g}$. In addition, this map preserves the subalgebras $\mathcal{Z}(\mathfrak{g})$ and $\mathcal{U}(\mathfrak{g})^{K}$. 
Definition 1. A distribution $T \in \mathcal{D}^{\prime}(G)$ is a fundamental solution of a differential operator $P \in \mathcal{U}(\mathfrak{g})$ if $P T=\delta$, where $\delta(f)=f(1)$; and $T$ is a parametrix of $P$ if $P T-\delta \in C^{\infty}(G)$.

In [5], Cerezo and Rouvière study the construction of parametrix and fundamental solutions in the group $G=S L(2, \mathbb{R})$ for operators in $\mathcal{U}(\mathfrak{g})^{K}$ (note that $\mathcal{U}(\mathfrak{g})^{K}$ is abelian in this case).

When $G$ has only one conjugacy class of Cartan subgroups ( $G$ complex semisimple, for example), in [13], Rouvière gives a sufficient condition for the existence of fundamental solution of bi-invariant operators (Theorem 4.2 of $[\mathbf{1 3}])$. He also proves a necessary condition for $P \in \mathcal{Z}(\mathfrak{g})$ to have a parametrix in an any connected semisimple group (Proposition 4.1 of [13]).

In this paper we extend the results of [5] and [13] for operators in $\mathcal{Z}\left(\mathcal{U}(\mathfrak{g})^{K}\right)$ to rank one groups and groups with one conjugacy class of Cartan subgroups.

If $G$ is Lie group with Lie algebra $\mathfrak{s} o(n, 1)$ or $\mathfrak{s} u(n, 1)$, it is a well know result that $\mathcal{U}(\mathfrak{g})^{K}$ is abelian, and isomorphic to $\mathcal{Z}(\mathfrak{g}) \otimes \mathcal{Z}(\mathfrak{k})$. This isn't true for other groups, but in general $\mathcal{Z}\left(\mathcal{U}(\mathfrak{g})^{K}\right) \simeq \mathcal{Z}(\mathfrak{g}) \otimes \mathcal{Z}(\mathfrak{k})$ (Knop's theorem [11]).

Given $\mathfrak{h}_{0}$ and $\mathfrak{t}_{0}$ Cartan subalgebras of $\mathfrak{g}_{0}$ and $\mathfrak{k}_{0}$ respectively, we will denote $\gamma_{\mathfrak{h}}^{G}$ and $\gamma_{\mathfrak{t}}^{K}$ the Harish-Chandra homomorphisms of $\mathcal{Z}(\mathfrak{g})$ and $\mathcal{Z}(\mathfrak{k})$ with respect to the subalgebras $\mathfrak{h}$ and $\mathfrak{t}$; then we have

$$
\begin{aligned}
& \mathcal{Z}(\mathfrak{g}) \times \mathcal{Z}(\mathfrak{k}) \stackrel{\gamma_{\mathfrak{h}}^{G} \times \gamma_{\mathfrak{t}}^{K}}{\longrightarrow} \mathcal{U}(\mathfrak{h})^{W} \times \mathcal{U}(\mathfrak{t})^{W_{K}} \\
& \downarrow \otimes \quad \downarrow \otimes \\
& \mathcal{Z}\left(\mathcal{U}(\mathfrak{g})^{K}\right) \stackrel{\simeq}{\longrightarrow} \mathcal{Z}(\mathfrak{g}) \otimes \mathcal{Z}(\mathfrak{k}) \stackrel{\gamma_{\mathfrak{h}}^{G} \otimes \gamma_{\mathfrak{t}}^{K}}{\longrightarrow} \mathcal{U}(\mathfrak{h})^{W} \otimes \mathcal{U}(\mathfrak{t})^{W_{K}} \stackrel{i \otimes i}{\longrightarrow} \mathcal{U}(\mathfrak{h} \oplus \mathfrak{t}) .
\end{aligned}
$$

Therefore, by the way of the homomorphisms described above, we can associate to $P \in \mathcal{Z}\left(\mathcal{U}(\mathfrak{g})^{K}\right)$ a differential operator $\left(\gamma_{\mathfrak{h}}^{G} \otimes \gamma_{\mathfrak{t}}^{K}\right)(P)$ in the group $H \times T$, where $H$ and $T$ are the respective Cartan subgroups of $G$ and $K$ with Lie algebras $\mathfrak{h}_{0}$ and $\mathfrak{t}_{0}$.

We say that $H^{f}$ is a fundamental Cartan subgroup of $G$ if $H^{f}$ has maximal compact factor between $\theta$-stable Cartan subgroups of $G$. All fundamental Cartan subgroups of $G$ are conjugate (c.f. [15], Chapter I).

We will denote $\gamma^{G}=\gamma_{\mathfrak{h}^{f}}^{G}$, where $\mathfrak{h}_{0}^{f}$ is the Lie subalgebra of a fundamental Cartan subgroup $H^{f}$. Because in $K$ all Cartan subgroups are conjugate, we put $\gamma^{K}=\gamma_{\mathfrak{t}}^{K}$.

We can now state our main result:

Theorem 1.1. Let $G$ be a connected semisimple Lie group of real rank one or with one conjugacy class of Cartan subgroups. Let $H^{f}$ be a fundamental Cartan subgroup of $G$ and $P \in \mathcal{Z}\left(\mathcal{U}(\mathfrak{g})^{K}\right)$. If $\left(\gamma^{G} \otimes \gamma^{K}\right)(P)$ has a fundamental solution in $H^{f} \times T$, then $P$ has a fundamental solution in $G$. 
When $P$ is a bi-invariant operator, we obtain a complete proof for these groups of the theorem announced in [3]:

Corollary 1.2 (Benabdallah-Rouvière). Let $P \in \mathcal{Z}(\mathfrak{g})$. If $\gamma^{G}(P)$ has a fundamental solution in $H^{f}$, then $P$ has a fundamental solution in $G$.

The proof will consist in the explicit construction of the fundamental solution of $P$, using the Plancherel formula as the main tool. When $G$ is a rank one group having a compact Cartan subgroup, using Zuckerman characters identities combined with the proof of 1.1, we obtain a sufficient condition for the existence of a parametrix of $P$ :

Theorem 1.3. Let $G$ be a linear connected semisimple Lie group of rank one such that $T$ is a compact Cartan subgroup of $G$, and $P \in \mathcal{Z}\left(\mathcal{U}(\mathfrak{g})^{K}\right)$. If $\left(\gamma^{G} \otimes \gamma^{K}\right)(P)$ has a parametrix on $T \times T$, then $P$ has a parametrix on $G$.

Finally we extend Proposition 4.1 of $[\mathbf{1 3}]$ for $P \in \mathcal{Z}\left(\mathcal{U}(\mathfrak{g})^{K}\right)$.

Acknowledgments. The results of this paper are part of my PhD-thesis. I wish to thank my advisor, Dr. Jorge Vargas, who suggested the problem and gave me continuous support and encouragement during these years. I also wish to thank Dr. Leandro Cagliero for many fruitful conversations about some topics of this paper, and particularly for his help with subsection 5.5.

Finally, I want to thank Professor Michel Duflo who gave a sketch for the proof of Proposition 6.3, which although I suppose well known, I couldn't find in the references.

\section{Preliminaries.}

In this section we fix notation and summarize the basics known facts about representation theory that will be needed through this paper.

2.1. Notation. Let $G$ be a connected reductive Lie group. $\theta$ will denote a Cartan involution in both $\mathfrak{g}_{0}$ and $G$. Let $\mathfrak{g}_{0}=\mathfrak{k}_{0} \oplus \mathfrak{p}_{0}$ be a Cartan decomposition of $\mathfrak{g}_{0}$ with respect to $\theta$; that is, $\mathfrak{k}_{0}=\left\{X \in \mathfrak{g}_{0}: \theta X=X\right\}$ and $\mathfrak{p}_{0}=\left\{X \in \mathfrak{g}_{0}: \theta X=-X\right\}$.

Let $K$ be analytic subgroup of $G$ with Lie algebra $\mathfrak{k}_{0}, K$ is a maximally compact subgroup of $G$. We fix $\mathfrak{t}_{0}$ a Cartan subalgebra of $\mathfrak{k}_{0}$ coming from a maximal torus $T$ of $K$. We will denote $\tau \in \hat{K}$ an irreducible unitary representation of $K$.

Let $\mathfrak{a}_{0}$ be a maximal abelian subalgebra of $\mathfrak{p}_{0}$. The dimension of $\mathfrak{a}_{0}$ is the real rank of $G$. We put $\mathfrak{m}_{0}=\left\{X \in \mathfrak{k}_{0}:\left[X, \mathfrak{a}_{0}\right]=0\right\}$. If $M=Z_{K}\left(\mathfrak{a}_{0}\right)=\{x \in$ $\left.K ;\left.\operatorname{Ad}(x)\right|_{\mathfrak{a}_{0}}=I d\right\}$, then $M$ is a compact subgroup of $G$ with Lie algebra $\mathfrak{m}_{0}$. If $\mathfrak{t}_{0}^{-}$is a Cartan subalgebra of $\mathfrak{m}_{0}$, then $\mathfrak{h}_{0}=\mathfrak{a}_{0} \oplus \mathfrak{t}_{0}^{-}$is a Cartan subalgebra of $\mathfrak{g}_{0}$.

If $B$ is the Killing form of $\mathfrak{g}_{0},\left.B\right|_{\mathfrak{k}_{0} \times \mathfrak{k}_{0}}$ is negative definite and $\left.B\right|_{\mathfrak{p}_{0} \times \mathfrak{p}_{0}}$ is positive definite. Then $(X, Y)=-B(X, \theta Y)$ defines an inner product on $\mathfrak{g}_{0}$. 
The complexification of any real Lie algebra will be denoted without the subscript. If $\mathfrak{g}_{u}=\mathfrak{k}_{0} \oplus i \mathfrak{p}_{0} \subseteq \mathfrak{g}$, then $\mathfrak{g}_{u}$ is a compact form of $\mathfrak{g}$. The Killing form of $\mathfrak{g}$ is the complex bilinear extension of that of $\mathfrak{g}_{0}$, and making an abuse of notation, we will still call it $B$. On $\mathfrak{g}$ we define the inner product, $(X, Y)=-B(X, J Y)$, where $J$ is conjugation with respect to $\mathfrak{g}_{u}$. Let's note that $\left.J\right|_{\mathfrak{g}_{0}}=\theta$ and so this inner product extends that of $\mathfrak{g}_{0}$.

Given $\sigma \in \hat{M}$ with infinitesimal character $\mu_{\sigma} \in i\left(\mathfrak{t}_{0}^{-}\right)^{\prime}$ and $\tau \in \hat{K}$ with infinitesimal character $\mu_{\tau} \in i \mathfrak{t}_{0}^{\prime}$, we define

$$
|\sigma|=\left|\mu_{\sigma}\right|, \quad|\tau|=\left|\mu_{\tau}\right| .
$$

We note that this definition makes sense because the norm is both $W_{M}$ and $W_{K}$ invariant, since the elements of the Weyl groups of $M$ and $K$ are inner automorphisms of $K$.

Every semisimple Lie group of rank one has at most two conjugacy classes of $\theta$-stable Cartan subgroups (or subalgebras). But in the case $G$ has only one, the hypothesis of rank one is not needed. So from now on we will restrict our attention to the following two cases:

I. $G$ has only one conjugacy class of $\theta$-stable Cartan subalgebras, which will be represented by $\mathfrak{h}_{0}=\mathfrak{a}_{0} \oplus \mathfrak{t}_{0}^{-}$.

II. $G$ is a rank one group having a compact Cartan subgroup. In this case $\mathfrak{t}_{0}$ is a Cartan subalgebra of $\mathfrak{g}_{0}$ and we can choose $\left\{\mathfrak{t}_{0}, \mathfrak{h}_{0}\right\}$ as a representative set of conjugacy classes of $\theta$-stable Cartan subalgebras.

We will also suppose that $G$ has a simply connected complexification $G^{\mathbb{C}}$. Finally we can assume, with no loss of generality, that $M$ is connected, because in case I, $M$ always is $([\mathbf{1 4}, 7.12 .7])$, and in case II, if $M$ is disconnected, then $G$ must be a direct product of $S L(2, \mathbb{R})$ with a compact group, and the results of this paper are easily deduced from [5] and [4].

2.2. Principal series. Let $\Lambda\left(\mathfrak{g}_{0}, \mathfrak{a}_{0}\right)$ be the restricted root system of $\mathfrak{g}_{0}$ with respect to $\mathfrak{a}_{0}$; we choose $\Lambda^{+}\left(\mathfrak{g}_{0}, \mathfrak{a}_{0}\right)$ a positive system and we put $\mathfrak{n}_{0}=\sum_{\lambda \in \Lambda^{+}} \mathfrak{g}_{0}^{\lambda}$. If $A$ and $N$ are the analytic subgroups with respective Lie algebras $\mathfrak{a}_{0}$ and $\mathfrak{n}_{0}$, then $M A N$ is a minimal parabolic subgroup of $G$.

Given $\sigma \in \hat{M}$ an irreducible representation of $M, \nu \in \mathfrak{a}^{\prime}$ a complex linear functional on $\mathfrak{a}$. The principal series representation $\pi_{\sigma, \nu}$ is defined inducing the representation $\sigma \otimes e^{\nu} \otimes 1$ from $M A N$ to $G$. The representation $\pi_{\sigma, \nu}$ is admissible; moreover the multiplicity of $\tau$ in $\left.\pi_{\sigma, \nu}\right|_{K}$ is independent of $\nu$ ([10, Prop. 8.4]) and will be denoted $n_{\tau}^{\sigma}$.

If $\mu_{\sigma} \in i \mathfrak{t}_{0}^{-}$is the infinitesimal character of $\sigma$ relative to $\mathfrak{t}^{-}$, then $\pi_{\sigma, \nu}$ has infinitesimal character $\mu_{\sigma}+\nu$ relative to $\mathfrak{a}+\mathfrak{t}^{-}$([10, Prop. 8.22]).

2.3. Discrete series (case II). Let $(\pi, V)$ be an irreducible unitary representation of $G$. We say that $(\pi, V)$ is a discrete series representation of $G$ if all its matrix coefficients $g \mapsto(\pi(g) u, v)$ are square integrable. $G$ admits 
discrete series representations if and only if $G$ has a compact Cartan subgroup; or equivalently, $\mathfrak{t}_{0}$ is a Cartan subalgebra of $\mathfrak{g}_{0}$ and $\mathfrak{k}_{0}$. All discrete series representations can be parametrized by the forms $\lambda \in i \mathbf{t}_{0}^{\prime}$ such that $\lambda$ is non-singular (i.e., $(\lambda, \alpha) \neq 0$ for all $\alpha \in \Delta(\mathfrak{g}, \mathfrak{t}))$ and $\lambda+\rho$ is analytically integral, or equivalently, $\lambda$ is integral, because we are assuming $G^{\mathbb{C}}$ simply connected. The functional $\lambda$ is called the Harish-Chandra parameter. Two discrete series representations are equivalent if and only if their parameters are conjugate by an element of the Weyl group $W_{K}$ of $K$. We will denote $\pi_{\lambda}$ the discrete series representation of parameter $\lambda$ and $\mathcal{S}_{d}$ the set of Harish-Chandra parameters.

The representation $\pi_{\lambda}$ has infinitesimal character $\lambda$, and discrete series representations with parameters $w \lambda, w \in W_{G}$, the Weyl group of $G$, have the same infinitesimal character. Therefore exactly $\left|W_{G}\right| /\left|W_{K}\right|$ of the representations $\pi_{w \lambda}$ are mutually inequivalent.

Finally, there is a positive number $d_{\lambda}$, called the formal degree of $\pi_{\lambda}$, such that

$$
\int_{G}\left(\pi_{\lambda}(x) u_{1}, v_{1}\right) \overline{\left(\pi_{\lambda}(x) u_{2}, v_{2}\right)} d x=d_{\lambda}^{-1}\left(u_{1}, u_{2}\right) \overline{\left(v_{1}, v_{2}\right)}
$$

for all $u_{1}, u_{2}, v_{1}, v_{2}$.

2.4. Global characters. We say that an admissible representation $\pi$ has a global character $\Theta_{\pi}$ if the operator

$$
\pi(f)=\int_{G} f(g) \pi(g) d g
$$

is a trace class operator for all $f \in \mathcal{D}(G)$ and if the map $f \mapsto \operatorname{tr} \pi(f)=\Theta_{\pi}(f)$ is a distribution on $G$.

Every admissible representation $\pi$ whose decomposition $\pi_{\mid K}=\sum_{\tau \in \hat{K}} n_{\tau} \tau$ satisfies $n_{\tau} \leq C \operatorname{dim} \tau$ has a global character ([10, Thm. 10.2]). As a consequence, every irreducible unitary representation has a character $([\mathbf{1 0}$, Thm. 8.1]). In the same way, induced representations from irreducible unitary representations also have characters. So discrete series as well as principal series representations have characters.

Given $\lambda \in \mathcal{S}_{d}$, we will denote $\Theta_{\lambda}$ the character of $\pi_{\lambda}$; and given $\sigma \in \hat{M}$, $\nu \in \mathfrak{a}^{\prime}, \Theta_{\sigma, \nu}$ the character of $\pi_{\sigma, \nu}$.

2.5. Plancherel formula. We are now in a position to write down the Plancherel formula for the groups we are considering (cf. [1, Lemma 5] and [14, Thm. 8.15.4]).

Theorem 2.1. There is a non-negative function $m_{\sigma}(\nu)$ defined in $\hat{M} \times i \mathfrak{a}_{0}^{\prime}$ such that for all $f \in \mathcal{D}(G)$ we have in case $\mathrm{I}$,

$$
f(1)=\sum_{\sigma \in \hat{M}} \int_{\nu \in i \mathfrak{a}_{0}^{\prime}} \Theta_{\sigma, \nu}(f) m_{\sigma}(\nu) d \nu,
$$


and in case II,

$$
f(1)=\sum_{\sigma \in \hat{M}} \int_{\nu \in i \mathfrak{a}_{0}^{\prime}} \Theta_{\sigma, \nu}(f) m_{\sigma}(\nu) d \nu+\sum_{\lambda \in \mathcal{S}_{d}} d_{\lambda} \Theta_{\lambda}(f) .
$$

The function $m_{\sigma}(\nu)$ has the following properties:

(i) For each $\sigma \in \hat{M}, m_{\sigma}(\nu)$ is the restriction to $i \mathfrak{a}_{0}^{\prime}$ of a meromorphic function on $\mathfrak{a}^{\prime}$ without poles on $i \mathfrak{a}_{0}^{\prime}$.

(ii) Exist a positive constant $C$ and a positive integer $l$ such that for all $\sigma \in \hat{M}, \nu \in i \mathfrak{a}_{0}^{\prime}$, we have

$$
\left|m_{\sigma}(\nu)\right| \leq C\left(1+|\sigma|^{2}\right)^{l}\left(1+|\nu|^{2}\right)^{l}
$$

\section{Action of $P$ on characters.}

If $\pi$ is an admissible representation with global character $\Theta_{\pi}$ and infinitesimal character $\chi_{\pi}$, and $P \in \mathcal{Z}(\mathfrak{g})$ is a bi-invariant differential operator, then $P \Theta_{\pi}=\chi_{\pi}(P) \Theta_{\pi}([\mathbf{1 0}$, Prop. 10.24]).

We want to prove a similar result for an operator $P \in \mathcal{Z}\left(\mathcal{U}(\mathfrak{g})^{K}\right)$. First we need to decompose a distribution as a sum of its $K$-isotypical components.

If we consider the right regular representation $R$ acting on $L^{2}(G)$ then $\mathcal{D}(G)$ is a dense subspace of $C^{\infty}$ vectors of $L^{2}(G)$, and decomposing $R$ in sum of its $K$-isotypical components we obtain

$$
f=\sum_{\tau \in \hat{K}} f_{\tau}
$$

where

$$
f_{\tau}(x)=d_{\tau}\left(f * \Theta_{\tau}\right)(x)=d_{\tau} \int_{K} f(x k) \Theta_{\tau}\left(k^{-1}\right) d k
$$

with $\Theta_{\tau}(k)=\operatorname{tr} \tau(k)$. Convergence holds not only in $L^{2}(G)$ but also in $\mathcal{D}(G)$. This decomposition induces a similar decomposition for a distribution $T \in \mathcal{D}^{\prime}(G)$ :

$$
T=\sum_{\tau \in \hat{K}} T^{\tau}
$$

with $T^{\tau}(f)=T\left(f_{\bar{\tau}}\right)$, where $\bar{\tau}$ is the conjugate representation of $\tau$. Remind that $\bar{\tau}$ is isomorphic to the contragredient representation $\tau^{*}$ of $K$.

Lemma 3.1. Let $(\pi, V)$ be an admissible representation. Suppose that its $K$-isotypical decomposition

$$
V=\sum_{\tau \in \hat{K}} n_{\tau} V_{\tau} \quad\left(L^{2} \mathrm{sum}\right)
$$

satisfies $n_{\tau} \leq C d_{\tau}$, where $d_{\tau}$ is the dimension of $\tau$, and $\left(\left.\pi\right|_{K}, V_{\tau}\right)$ is equivalent to $\tau$. We choose an orthonormal base of $V$ by joining orthonormal bases $\left\{e_{i}^{\tau}: i=1 \ldots d_{\tau}\right\}$ of $V_{\tau}$. 
If $\Theta_{\pi}$ is the character of $\pi$, then:

$$
\Theta_{\pi}^{\tau}(f)=n_{\tau} \sum_{i=1}^{d_{\tau}}\left(\pi(f) e_{i}^{\tau}, e_{i}^{\tau}\right)
$$

Proof.

$$
\Theta_{\pi}^{\tau}(f)=\Theta_{\pi}\left(f_{\bar{\tau}}\right)=\sum_{\tau \in \hat{K}} n_{\tau}\left[\sum_{i=1}^{d_{\tau}}\left(\pi\left(f_{\bar{\tau}}\right) e_{i}^{\tau}, e_{i}^{\tau}\right)\right]
$$

with

$$
f_{\bar{\tau}}=d_{\tau}\left(f * \Theta_{\bar{\tau}}\right)=d_{\tau}\left(f * \bar{\Theta}_{\tau}\right),
$$

where $\Theta_{\tau}$ is a $C^{\infty}$ function on $K$ being $\tau$ of finite dimension.

Let $p_{\tau}: V \longrightarrow V_{\tau}$ be the orthogonal projection. Now let $\tau^{\prime} \in \hat{K}$ be another representation; then, using Fubini's theorem and the bi-invariance of the Haar measures of $G$ and $K$,

$$
\begin{aligned}
& \left(\pi\left(f_{\bar{\tau}}\right) e_{i}^{\tau^{\prime}}, e_{i}^{\tau^{\prime}}\right) \\
& =\int_{G} f_{\bar{\tau}}(x)\left(\pi(x) e_{i}^{\tau^{\prime}}, e_{i}^{\tau^{\prime}}\right) d x \\
& =\int_{G}\left[d_{\tau} \int_{K} f(x k) \bar{\Theta}_{\tau}\left(k^{-1}\right) d k\right]\left(\pi(x) e_{i}^{\tau^{\prime}}, e_{i}^{\tau^{\prime}}\right) d x \\
& =\int_{G} f(x)\left[d_{\tau} \int_{K}\left(\pi\left(k^{-1}\right) e_{i}^{\tau^{\prime}}, \pi(x)^{*} e_{i}^{\tau^{\prime}}\right) \bar{\Theta}_{\tau}\left(k^{-1}\right) d k\right] d x \\
& =\int_{G} f(x)\left[\sum_{j=1}^{d_{\tau}} d_{\tau} \int_{K}\left(\pi(k) e_{i}^{\tau^{\prime}}, p_{\tau^{\prime}}\left(\pi(x)^{*} e_{i}^{\tau^{\prime}}\right)\right) \overline{\left(\tau(k) e_{j}^{\tau}, e_{j}^{\tau}\right)} d k\right] d x
\end{aligned}
$$

and by Schur orthogonality relations (Corollary 1.10 of [10]),

$$
\begin{aligned}
\left(\pi\left(f_{\bar{\tau}}\right) e_{i}^{\tau^{\prime}}, e_{i}^{\tau^{\prime}}\right) & =\int_{G} f(x)\left[\sum_{j=1}^{d_{\tau}} \delta_{\tau, \tau^{\prime}}\left(e_{i}^{\tau^{\prime}}, e_{j}^{\tau}\right) \overline{\left(p_{\tau^{\prime}}\left(\pi(x)^{*} e_{i}^{\tau^{\prime}}\right), e_{j}^{\tau}\right)} d k\right] d x \\
& =\delta_{\tau, \tau^{\prime}} \int_{G} f(x) \overline{\left(\pi(x)^{*} e_{i}^{\tau^{\prime}}, e_{i}^{\tau^{\prime}}\right)} d x \\
& =\delta_{\tau, \tau^{\prime}} \int_{G} f(x)\left(\pi(x) e_{i}^{\tau}, e_{i}^{\tau}\right) d x
\end{aligned}
$$

Given $\pi$ a representation of $G, \tau \in \hat{K}$, the map $\chi_{\pi} \otimes \chi_{\tau}$ is a linear functional on $\mathcal{Z}(\mathfrak{g}) \otimes \mathcal{Z}(\mathfrak{k})$, and induces a linear functional on $\mathcal{Z}\left(\mathcal{U}(\mathfrak{g})^{K}\right)$, which we will still denote $\chi_{\pi} \otimes \chi_{\tau}$.

We can now state the result we need. 
Proposition 3.2. If $\pi$ is an admissible representation with infinitesimal character $\chi_{\pi}$ and global character $\Theta_{\pi}$, and $P$ is a differential operator in $\mathcal{Z}\left(\mathcal{U}(\mathfrak{g})^{K}\right)$, then

$$
P \Theta_{\pi}^{\tau}=\left(\chi_{\pi} \otimes \chi_{\tau}\right)(P) \Theta_{\pi}^{\tau} .
$$

Proof. We preserve the notation of Lemma 3.1. If $P \in \mathcal{Z}(\mathfrak{g}), P \Theta_{\pi}^{\tau}=$ $\chi_{\pi}(P) \Theta_{\pi}^{\tau}$. If $P \in \mathcal{Z}(\mathfrak{k})$,

$$
P \Theta_{\pi}^{\tau}(f)=\Theta_{\pi}^{\tau}\left(P^{t} f\right)=n_{\tau} \sum_{i=1}^{d_{\tau}}\left(\pi\left(P^{t} f\right) e_{i}^{\tau}, e_{i}^{\tau}\right)
$$

and

$$
\begin{aligned}
\left(\pi\left(P^{t} f\right) e_{i}^{\tau}, e_{i}^{\tau}\right) & =\int_{G}\left(\pi(x) \pi(P) e_{i}^{\tau}, e_{i}^{\tau}\right) f(x) d x \\
& =\chi_{\tau}(P)\left(\pi(f) e_{i}^{\tau}, e_{i}^{\tau}\right) \quad \text { by }[\mathbf{1 0},(8.10)],
\end{aligned}
$$

because $\left.\pi(P)\right|_{V_{\tau}}=\tau(P)$. Then $P \Theta_{\pi}^{\tau}=\chi_{\tau}(P) \Theta_{\pi}^{\tau}$ if $P \in \mathcal{Z}(\mathfrak{k})$.

\section{Fundamental solutions on abelian connected groups.}

In [4], Cerezo and Rouvière obtain necessary and sufficient conditions for $P \in \mathcal{U}(\mathfrak{g})$ to have a fundamental solution when $G$ is a connected compact group or a product of a connected compact group with $\mathbb{R}^{n}$.

In this section we state these results for a connected abelian group, which is the product of a torus with $\mathbb{R}^{n}$. Theorem 4.1 follows directly from Theorem III of $[4]$.

We consider in first place an abelian connected compact Lie group $T$ with Lie algebra $\mathfrak{t}_{0}$, i.e., $T$ a torus. In this case $\exp : \mathfrak{t}_{0} \longrightarrow T$ is a group epimorphism, and $\Gamma=\operatorname{ker}(\exp )$ is a closed discrete subgroup of $\mathfrak{t}_{0}$. Being $T$ abelian, its irreducible unitary representations are one dimensional and can be parametrized by the linear functionals $\lambda \in i \mathfrak{t}_{0}^{\prime}$ such that $\lambda(\Gamma) \subseteq 2 \pi i \mathbb{Z}$. So we set

$$
\hat{T}=\left\{\lambda \in i \mathfrak{t}_{0}^{\prime} \text { such that } \lambda(\Gamma) \subseteq 2 \pi i \mathbb{Z}\right\} .
$$

A general abelian connected Lie group is of the form $A \times T$, where $A=\mathbb{R}^{n}$ and $T$ is a $m$-dimensional torus. The universal algebra $\mathcal{U}(\mathfrak{a} \oplus \mathfrak{t})$ coincides with the symmetric algebra $\mathcal{S}(\mathfrak{a} \oplus \mathfrak{t})$, and so an element $P$ in $\mathcal{U}(\mathfrak{a} \oplus \mathfrak{t})$ can be thought of both as a constant coefficient differential operator on $A \times T$ and as a polynomial function on $\mathfrak{a}^{\prime} \oplus \mathfrak{t}^{\prime}$. Given $\lambda \in \hat{T}$, if we put

$$
P_{\lambda}(x)=P(x, \lambda), x \in \mathfrak{a}^{\prime},
$$

then $P_{\lambda}$ is a polynomial function in $\mathbb{R}^{n}$, and its norm can be defined

$$
\left\|P_{\lambda}\right\|=\left(\sum_{\alpha} \frac{1}{(\alpha !)^{2}}\left|P_{\lambda}^{(\alpha)}(0)\right|^{2}\right)^{\frac{1}{2}} .
$$


Theorem 4.1. Let $P \in \mathcal{U}(\mathfrak{a} \oplus \mathfrak{t})$ be a constant coefficients differential operator on $A \times T . P$ has a fundamental solution if and only if exists a positive constant $C$ and a positive integer $k$ such that in any norm of $i \mathbf{t}_{0}^{\prime}$

$$
\left\|P_{\lambda}\right\| \geq \frac{C}{\left(1+|\lambda|^{2}\right)^{k}} \quad \forall \lambda \in \hat{T} .
$$

Remark. Let's go back to $G$ a connected semisimple Lie group. Remember that given $P \in \mathcal{Z}\left(\mathcal{U}(\mathfrak{g})^{K}\right)$ and $\mathfrak{h}_{0}$ and $\mathfrak{t}_{0}$ Cartan subalgebras of $\mathfrak{g}_{0}$ and $\mathfrak{k}_{0}$ respectively we have defined a differential operator $\left(\gamma_{\mathfrak{h}}^{G} \otimes \gamma_{\mathfrak{t}}^{K}\right)(P)$ on the group $H \times T$. This operator depends on the choice of the Cartan subgroups of $G$ and $K$. However, the existence of fundamental solution only depends on the conjugacy class (in $G$ or $K$ ) of Cartan subgroups, as we see below.

Let $\tilde{H}$ and $\tilde{T}$ Cartan subgroups of $G$ and $K$ respectively and suppose there exist $g \in G, k \in K$ such that $\tilde{H}=g^{-1} H g$ and $\tilde{T}=k^{-1} T k$. Then $\operatorname{Ad}(g) \mathfrak{h}_{0}=\tilde{\mathfrak{h}}_{0}$ and Ad $(k) \mathfrak{t}_{0}=\tilde{\mathfrak{t}}_{0}$. If $H=A \times T^{-}, \mathfrak{h}_{0}=\mathfrak{a}_{0}+\mathfrak{t}_{0}$, then $\tilde{H}=\tilde{A} \times \widetilde{T^{-}}, \tilde{A}=\operatorname{Ad}(g) A, \widetilde{T^{-}}=\operatorname{Ad}(g) T^{-}$, and is clear from (4) that

$$
\widehat{\tilde{T}}=\left\{\tilde{\lambda}=\lambda \circ \operatorname{Ad}(g)^{-1}: \lambda \in \hat{T}\right\}, \quad \widehat{T^{-}}=\left\{\tilde{\mu}=\mu \circ \operatorname{Ad}(k)^{-1}: \mu \in \widehat{T^{-}}\right\} ;
$$

besides we have

$$
\gamma_{\tilde{\mathfrak{h}}}^{G}=\operatorname{Ad}(g) \circ \gamma_{\mathfrak{h}}^{G}, \quad \gamma_{\tilde{\mathfrak{t}}}^{K}=\operatorname{Ad}(k) \circ \gamma_{\mathfrak{h}}^{K},
$$

so if $x \in \mathfrak{a}^{\prime}, \tilde{x}=x \circ \operatorname{Ad}(g)^{-1} \in \tilde{\mathfrak{a}}^{\prime},(\tilde{\lambda}, \tilde{\mu}) \in \widehat{\tilde{T}} \times \widehat{\widehat{T^{-}}}$and if $P \in \mathcal{Z}\left(\mathcal{U}(\mathfrak{g})^{K}\right)$,

$$
\begin{aligned}
P_{(\tilde{\lambda}, \tilde{\mu})}(\tilde{x}) & =\left(\left(\gamma_{\tilde{\mathfrak{h}}}^{G} \otimes \gamma_{\tilde{\mathfrak{t}}}^{K}\right)(P)\right)(\tilde{x}, \tilde{\lambda}, \tilde{\mu}) \\
& =\left(\left(\gamma_{\mathfrak{h}}^{G} \otimes \gamma_{\mathfrak{t}}^{K}\right)(P)\right)(x, \lambda, \mu)=P_{(\lambda, \mu)}(x),
\end{aligned}
$$

and so Theorem 4.1 implies that $\left(\gamma_{\mathfrak{h}}^{G} \otimes \gamma_{\mathfrak{t}}^{K}\right)(P)$ has a fundamental solution on $H \times T$ if and only if $\left(\gamma_{\tilde{\mathfrak{h}}}^{G} \otimes \gamma_{\tilde{\mathfrak{t}}}^{K}\right)(P)$ has one on $\tilde{H} \times \tilde{T}$.

\section{Inversion of infinitesimal characters.}

5.1. Case I. Let $\mathfrak{h}_{0}=\mathfrak{a}_{0}+\mathfrak{t}_{0}^{-}$be a Cartan subalgebra of $\mathfrak{g}_{0}$, where $\mathfrak{t}_{0}^{-}$is a Cartan subalgebra of $\mathfrak{m}_{0}$, let $\mathfrak{t}_{0}$ be a Cartan subalgebra of $\mathfrak{k}_{0}$. Let $\sigma \in \hat{M}$ with infinitesimal character $\mu_{\sigma} \in i\left(\mathfrak{t}_{0}^{-}\right)^{\prime}, \nu \in \mathfrak{a}^{\prime}, \tau \in \hat{K}$ with infinitesimal character $\mu_{\tau} \in i \mathfrak{t}_{0}^{\prime}$.

Recall that to an operator $P \in \mathcal{Z}\left(\mathcal{U}(\mathfrak{g})^{K}\right)$ we associate an operator $\left(\gamma_{\mathfrak{h}}^{G} \otimes \gamma^{K}\right)(P)$ on the abelian connected group $A \times T^{-} \times T$. We then have

$$
\left(\chi_{\sigma, \nu} \otimes \chi_{\tau}\right)(P)=\left(\left(\gamma_{\mathfrak{h}}^{G} \otimes \gamma^{K}\right)(P)\right)\left(\nu+\mu_{\sigma}, \mu_{\tau}\right),
$$


and fixing $\sigma, \tau$ we obtain a polynomial function in $\mathfrak{a}^{\prime}$, and we put

$$
P_{\sigma, \tau}(\nu)=\left(\chi_{\sigma, \nu} \otimes \chi_{\tau}\right)(P) \text {. }
$$

Proposition 5.1. Given $P \in \mathcal{Z}\left(\mathcal{U}(\mathfrak{g})^{K}\right)$, if $\left(\gamma_{\mathfrak{h}}^{G} \otimes \gamma^{K}\right)(P)$ has a fundamental solution on $A \times T^{-} \times T$ there exist a constant $C$ and a positive integer $k$ such that

$$
\left\|P_{\sigma, \tau}\right\| \geq \frac{C}{\left(1+|\sigma|^{2}\right)^{k}\left(1+|\tau|^{2}\right)^{k}} \quad \forall(\sigma, \tau) \in \hat{M} \times \hat{K} .
$$

Proof. According to (6) and (9), $P_{\sigma, \tau}(\nu)=\left(\left(\gamma_{\mathfrak{h}}^{G} \otimes \gamma^{K}\right)(P)\right)_{\left(\mu_{\sigma}, \mu_{\tau}\right)}(\nu) ; \mu_{\sigma}$ as well as $\mu_{\tau}$ are analytically integral forms, so $\left(\mu_{\sigma}, \mu_{\tau}\right) \in \widehat{T^{-}} \times \hat{T}$, and the proposition follows directly from Theorem 4.1.

5.2. Case II: Discrete series. Let $\mathfrak{t}_{0}$ be a Cartan subalgebra of $\mathfrak{g}_{0}$ and $\mathfrak{k}_{0}$. Let $\pi_{\lambda}$ be the discrete series representation with parameter $\lambda \in i \mathfrak{t}_{0}^{\prime}$, and $\tau \in \hat{K}$ with infinitesimal character $\mu_{\tau} \in i \mathfrak{t}_{0}^{\prime}$.

In this case, given $P \in \mathcal{Z}\left(\mathcal{U}(\mathfrak{g})^{K}\right),\left(\gamma_{\mathfrak{t}}^{G} \otimes \gamma^{K}\right)(P)$ is a differential operator on $T \times T$, and we put

$$
P_{\lambda, \tau}=\left(\chi_{\lambda} \otimes \chi_{\tau}\right)(P)=\left(\gamma_{\mathfrak{t}}^{G} \otimes \gamma^{K}(P)\right)\left(\lambda, \mu_{\tau}\right) .
$$

Proposition 5.2. Given $P \in \mathcal{Z}\left(\mathcal{U}(\mathfrak{g})^{K}\right)$, if $\left(\gamma_{\mathfrak{t}}^{G} \otimes \gamma^{K}\right)(P)$ has a fundamental solution on $T \times T$, there exist a constant $C$ and a positive integer $k$ such that

$$
\left|P_{\lambda, \tau}\right| \geq \frac{C}{\left(1+|\lambda|^{2}\right)^{k}\left(1+|\tau|^{2}\right)^{k}} \quad \forall(\lambda, \tau) \in \mathcal{S}_{d} \times \hat{K} .
$$

As in Proposition 5.1, the proof is a direct consequence of Theorem 4.1 with $A=0$, observing that $\left(\lambda, \mu_{\tau}\right) \in \hat{T} \times \hat{T}$.

5.3. Case II: Principal series. In this case we need to invert simultaneously the infinitesimal characters of principal and discrete series representations. We will prove the following:

Proposition 5.3. Given $P \in \mathcal{Z}\left(\mathcal{U}(\mathfrak{g})^{K}\right)$, suppose that for a finite set $F$ exist a constant $C$ and a positive integer $k$ such that

$$
\left|P_{\lambda, \tau}\right| \geq \frac{C}{\left(1+|\lambda|^{2}\right)^{k}\left(1+|\tau|^{2}\right)^{k}} \quad \forall(\lambda, \tau) \in\left(\mathcal{S}_{d}-F\right) \times \hat{K} .
$$

Then there exist a constant $\tilde{C}$ and a positive integer $\tilde{k}$ such that

$$
\left\|P_{\sigma, \tau}\right\| \geq \frac{\tilde{C}}{\left(1+|\sigma|^{2}\right)^{\tilde{k}}\left(1+|\tau|^{2}\right)^{\tilde{k}}} \quad \forall(\sigma, \tau) \in \hat{M} \times \hat{K} .
$$

For this purpose we will introduce the Cayley transform which is an inner automorphism of the complex group $G^{\mathbb{C}}$ that conjugates the Cartan subalgebras $\mathfrak{t}$ and $\mathfrak{h}$. 
5.4. Cayley transform. Given a compact Cartan subgroup $T$ with Lie algebra $\mathfrak{t}_{0}$, and given a non-compact root $\beta \in \Delta(\mathfrak{g}, \mathfrak{t})$, we can construct a Cayley transform $\mathbf{c}_{\beta}$ and a non-compact Cartan subgroup $H$ in the following way:

Let $H_{\beta} \in \mathfrak{t}$ such that $\beta(H)=B\left(H, H_{\beta}\right)$ for all $H \in \mathfrak{t}$ and we put $H_{\beta}^{\prime}=$ $2|\beta|^{-2} H_{\beta}$. We choose root vectors $X_{\beta}^{\prime} \in \mathfrak{g}_{\beta}$ and $X_{-\beta}^{\prime}=-\theta X_{\beta}^{\prime} \in \mathfrak{g}_{-\beta}$ such that $B\left(X_{\beta}^{\prime}, X_{-\beta}^{\prime}\right)=2|\beta|^{-2}$ and such that $X_{\beta}^{\prime}+X_{-\beta}^{\prime}$ and $i\left(X_{\beta}^{\prime}-X_{-\beta}^{\prime}\right)$ are in $\mathfrak{g}_{0}$. We then define

$$
\mathbf{c}_{\beta}=\operatorname{Ad}\left(\exp \frac{\pi}{4}\left(X_{-\beta}^{\prime}-X_{\beta}^{\prime}\right)\right)
$$

and

$$
\mathfrak{h}_{0}=\mathfrak{g}_{0} \cap \mathbf{c}_{\beta}(\mathfrak{t}) .
$$

If we put $\operatorname{ker} \beta=\left\{H \in \mathfrak{t}_{0}: \beta(H)=0\right\}$, then $\mathfrak{t}_{0}=\operatorname{ker} \beta \oplus \mathbb{R} i H_{\beta}$. Now $\left.\mathbf{c}_{\beta}\right|_{\operatorname{ker} \beta}=I d$ and $\mathbf{c}_{\beta}\left(i H_{\beta}\right)=i\left(X_{\beta}^{\prime}+X_{-\beta}^{\prime}\right)$, so

$$
\mathfrak{h}_{0}=\operatorname{ker} \beta \oplus \mathbb{R}\left(X_{\beta}^{\prime}+X_{-\beta}^{\prime}\right), \quad \mathbf{c}_{\beta}\left(\mathfrak{t}_{0}\right)=\operatorname{ker} \beta \oplus \mathbb{R} i\left(X_{\beta}^{\prime}+X_{-\beta}^{\prime}\right) .
$$

Let $\mathfrak{a}_{0}=\mathbb{R}\left(X_{\beta}^{\prime}+X_{-\beta}^{\prime}\right), \mathfrak{a}_{0}$ is a maximal abelian subalgebra in $\mathfrak{p}_{0}$ because $G$ is of rank one. Besides $\mathfrak{m}_{0}=\left\{X \in \mathfrak{g}_{0}:\left[X, \mathfrak{a}_{0}\right]=0\right\}$, and it's clear by the choice of $\mathfrak{a}_{0}$ that $\operatorname{ker} \beta \subset \mathfrak{m}_{0}$, and $\mathfrak{t}_{0}^{-}=\operatorname{ker} \beta$ is maximal abelian subalgebra of $\mathfrak{m}_{0}$ by dimension. So $\mathbf{c}_{\beta}$ carries $\mathfrak{t}$ on $\mathfrak{h}$ fixing $\mathfrak{t}^{-}$.

\subsection{Extension of infinitesimal characters of representations of $\mathbf{M}$.}

If we fix a positive root system $\Delta^{+}(\mathfrak{g}, \mathfrak{t})$, the set of infinitesimal characters of $\mathcal{S}_{d}$ can be parametrized by the set of strongly dominant integral forms, that is,

$$
\left\{\lambda \in i \mathfrak{t}_{0}^{\prime}: \lambda\left(\Gamma_{T}\right) \subseteq 2 \pi i \mathbb{Z} \text { and }(\lambda, \alpha)>0 \forall \alpha \in \Delta^{+}(\mathfrak{g}, \mathfrak{t})\right\},
$$

where $\Gamma_{T}=\operatorname{ker}\left(\left.\exp \right|_{\mathfrak{t}_{0}}\right)$. Now, being $\mathfrak{t}_{0}$ a Cartan subalgebra of $\mathfrak{g}_{u}$, this set coincides with the set of infinitesimal characters of irreducible unitary representations of finite dimension of the compact form $G_{u}$ of $G$.

Note that $\mathfrak{h}_{u}=\mathbf{c}_{\beta}\left(\mathfrak{t}_{0}\right)=i \mathfrak{a}_{0} \oplus \mathfrak{t}_{0}^{-}$is another Cartan subalgebra of $\mathfrak{g}_{u}$, and we can also parametrize the set of infinitesimal characters of $\widehat{G}_{u}$, and then that of $\mathcal{S}_{d}$, with the set

$$
\left\{\tilde{\lambda} \in\left(\mathfrak{a}_{0} \oplus i \mathfrak{t}_{0}^{-}\right)^{\prime}: \tilde{\lambda}\left(\Gamma_{H_{u}}\right) \subseteq 2 \pi i \mathbb{Z} \text { and }(\tilde{\lambda}, \tilde{\alpha})>0 \forall \tilde{\alpha} \in \Delta^{+}(\mathfrak{g}, \mathfrak{h})\right\},
$$

where $\Gamma_{H_{u}}=\operatorname{ker}\left(\left.\exp \right|_{\mathfrak{h} u}\right)=\mathbf{c}_{\beta}\left(\Gamma_{T}\right)$; putting $\tilde{\lambda}=\mathbf{c}_{\beta}(\lambda)=\lambda \circ \mathbf{c}_{\beta}^{-1}$, it's clear that $\mathbf{c}_{\beta}$ provides a bijection between both sets.

Besides, if $\Gamma_{T^{-}}=\operatorname{ker}\left(\left.\exp \right|_{\mathfrak{t}_{0}^{-}}\right)$, the set of infinitesimal characters of irreducible unitary representations of $M$ is given by

$$
\left\{\mu \in i\left(\mathfrak{t}_{0}^{-}\right)^{\prime}: \mu\left(\Gamma_{T^{-}}\right) \subseteq 2 \pi i \mathbb{Z} \text { and }(\mu, \alpha)>0 \forall \alpha \in \Delta^{+}\left(\mathfrak{m}, \mathfrak{t}^{-}\right)\right\} .
$$


For any $\lambda \in \mathcal{S}_{d}$, if we put $\mu=\left.\mathbf{c}_{\beta}(\lambda)\right|_{\mathfrak{t}_{0}^{-}}$, then

$$
\mu\left(\Gamma_{T^{-}}\right)=\lambda\left(\mathbf{c}_{\beta}^{-1} \Gamma_{T^{-}}\right) \subseteq \lambda\left(\Gamma_{T}\right) \subseteq 2 \pi i \mathbb{Z},
$$

and if $\alpha \in \Delta\left(\mathfrak{m}, \mathfrak{t}^{-}\right), H_{\alpha} \in i \mathfrak{t}_{0}^{-} \subseteq i \mathfrak{t}_{0}$, by suitable choice of the respective positive systems,

$$
(\mu, \alpha)=\mu\left(H_{\alpha}\right)=\lambda\left(\mathbf{c}_{\beta}^{-1} H_{\alpha}\right)=\lambda\left(H_{\alpha}\right)=(\lambda, \alpha)>0,
$$

and there exists $\sigma \in \hat{M}$ such that $\mu_{\sigma}=\left.\mathbf{c}_{\beta}(\lambda)\right|_{\mathfrak{t}_{0}^{-}}$. We want to see that every infinitesimal character $\mu_{\sigma}$ can be obtained in this way.

Proposition 5.4. Given $F$ a finite subset of $\mathcal{S}_{d}$, for all $\sigma \in \hat{M}$ exists a discrete series parameter $\lambda \in \mathcal{S}_{d}-F$ such that $\mu_{\sigma}=\left.\mathbf{c}_{\beta}(\lambda)\right|_{\mathfrak{t}_{0}^{-}}$. Moreover, $\lambda$ can be chosen such that $|\lambda| \leq C|\mu|$ for some constant $C$ (independent of $\mu$ ).

For the proof we will need some previous lemmas.

We choose positive root systems in $\mathfrak{g}$ and $\mathfrak{m}$ in the following way: Let $\left\{H_{1}, \ldots, H_{n}\right\}$ be an ordered basis of $\mathfrak{a}_{0}+i \mathfrak{t}_{0}^{-}$such that $H_{1}$ is a basis of $\mathfrak{a}_{0}$ and $\left\{H_{2}, \ldots, H_{n}\right\}$ is basis of $i \mathfrak{t}_{0}^{-}$and let $\Delta^{+}(\mathfrak{g}, \mathfrak{h})$ be the respective positive root system. Then

$$
\Delta^{+}\left(\mathfrak{m}, \mathfrak{t}^{-}\right)=\left\{\alpha \in \Delta^{+}(\mathfrak{g}, \mathfrak{h}):\left.\alpha\right|_{\mathfrak{a}_{0}}=0\right\} .
$$

We also choose $\Delta^{+}(\mathfrak{g}, \mathfrak{t})$ as the image of $\Delta^{+}(\mathfrak{g}, \mathfrak{h})$ by $\mathbf{c}_{\beta}^{-1}$.

Let $\Lambda^{+}\left(\mathfrak{g}_{0}, \mathfrak{a}_{0}\right)=\left\{\left.\alpha\right|_{\mathfrak{a}_{0}}: \alpha \in \Delta^{+}(\mathfrak{g}, \mathfrak{h})\right\}$ be the respective positive restricted root system. Because of the rank one condition, there exists $\beta_{0} \in \mathfrak{a}_{0}^{\prime}$ such that $\Lambda^{+}\left(\mathfrak{g}_{0}, \mathfrak{a}_{0}\right)=\left\{\beta_{0}\right\}$ or $\Lambda^{+}\left(\mathfrak{g}_{0}, \mathfrak{a}_{0}\right)=\left\{\frac{1}{2} \beta_{0}, \beta_{0}\right\}$; and we will still denote $\beta_{0}$ its extension to $\left(\mathfrak{a}_{0}+i \mathfrak{t}_{0}^{-}\right)^{\prime}$ by 0 in $i\left(\mathfrak{t}_{0}^{-}\right)^{\prime}$.

Lemma 5.5. $\beta_{0}$ belongs to the positive root system $\Delta^{+}(\mathfrak{g}, \mathfrak{h})$.

Proof. Follows directly from Lemmas 1 and 2, p. 33 of [15].

Lemma 5.6. If $\left\{\alpha_{1}, \ldots, \alpha_{n}\right\}$ is a simple root system of $\mathfrak{g}$ with respect to the positive system fixed previously, there exist at most two simple roots such that their restriction to $\mathfrak{a}_{0}$ are not identically 0 .

Proof. Let $\alpha=m_{1} \alpha_{1}+\cdots+m_{n} \alpha_{n}$ be the maximal positive root of $\Delta^{+}(\mathfrak{g}, \mathfrak{h})$; that is, $m_{i} \in \mathbb{N}$ for all $i$. Now $\left.\alpha_{i}\right|_{\mathfrak{a}_{0}}=s_{i} \beta_{0}$, con $s_{i}=0,1 / 2$ or 1 for all $i$, therefore $m_{1} s_{1}+\cdots+m_{n} s_{n} \leq 1$ and so at most two of the $s_{i}$ can be 0 .

Lemma 5.7. Let $G$ be a semisimple rank one Lie group. For every $\mu \in$ $\left(i \mathfrak{t}_{0}^{-}\right)^{\prime}$ strongly dominant analytically integral form, exists $k \in \mathbb{R}$ such that $k \beta_{0}+\mu$ is strongly dominant integral in $\left(\mathfrak{a}_{0}+i \mathfrak{t}_{0}^{-}\right)^{\prime}$. Moreover, we can choose $k$ with the following properties:

(i) $k \beta_{0}+\mu \notin F$, where $F$ is a fixed finite subset of strongly dominant integral forms in $\left(\mathfrak{a}_{0}+i \mathfrak{t}_{0}^{-}\right)^{\prime}$.

(ii) $|k| \leq C|\mu|$ for some constant $C$ (independent of $\mu$ ). 
Proof. With no loss of generality, we can suppose that $\mathfrak{g}$ is simple.

Let $\left\{\alpha_{1}, \ldots, \alpha_{n}\right\}$ be a simple root system of $\mathfrak{g}$ with respect to the positive system previously chosen. Let $\left\{\lambda_{1}, \ldots, \lambda_{n}\right\}$ be the fundamental weights of $\mathfrak{g}$ with respect to to this simple root system.

Then $\left\{\alpha_{i}:\left.\alpha_{i}\right|_{\mathfrak{a}_{0}}=0\right\}$ is a simple root system of the semisimple part of $\mathfrak{m}$. If $\lambda_{i}^{M}$ is the respective fundamental weight in $\mathfrak{m}$ and we extend it by 0 to $\left(\mathfrak{a}_{0}+i \mathfrak{t}_{0}^{-}\right)^{\prime}$,

$$
\lambda_{i}=\lambda_{i}^{M}+k_{i} \beta_{0}
$$

because $\lambda_{i}-\left.\lambda_{i}^{M}\right|_{\mathfrak{t}_{0}^{-}}=0$, and $k_{i}=\frac{\left(\lambda_{i}, \beta_{0}\right)}{\left(\beta_{0}, \beta_{0}\right)} \in \frac{1}{2} \mathbb{Z}$ being $\beta_{0}$ a root. We will analyze two cases:

I) The simple root $\alpha_{1}$ is the only one with non-vanishing restriction to $\mathfrak{a}_{0}$. In this case, $M$ is semisimple. Now, because $\left(\lambda_{1}, \alpha_{i}\right)=0$ for all $i \geq 2$, $\left.\lambda_{1}\right|_{\mathfrak{t}_{0}^{-}}=0$ then $\lambda_{1}=k_{1} \beta_{0}$, with $k_{1} \in \frac{1}{2} \mathbb{Z}$.

So, if $\mu$ is strongly dominant integral, $\mu=m_{2} \lambda_{2}^{M}+\cdots+m_{n} \lambda_{n}^{M}$ with $m_{i} \in \mathbb{N}$ for all $i \geq 2$ and

$k \beta_{0}+\mu=\left(k-\sum_{i=2}^{n} m_{i} k_{i}\right) \beta_{0}+\sum_{i=2}^{n} m_{i} \lambda_{i}=\left(k-\sum_{i=2}^{n} m_{i} k_{i}\right) \frac{1}{k_{1}} \lambda_{1}+\sum_{i=2}^{n} m_{i} \lambda_{i}$,

so it's enough to choose $k=k_{0} k_{1}+m_{2} k_{2}+\cdots+m_{n} k_{n} \in \frac{1}{2} \mathbb{Z}$ with $k_{0} \in \mathbb{Z}^{+}$ minimum subject to the condition $k \beta_{0}+\mu \notin F$.

II) There exist two simple roots with non-vanishing restriction to $\mathfrak{a}_{0}$. In this case, looking at the Satake diagrams ([15, Chap. 1], it's clear that the only possibility is $\mathfrak{g}_{0} \simeq \mathfrak{s} u(n, 1)$. We choose $\mathfrak{h}_{0}$ as the 0 trace diagonal matrices. If $H=\sum i h_{i} E_{i i}$, let $e_{i} \in i \mathfrak{h}_{0}^{\prime}$ be defined by $e_{i}(H)=h_{i}$. We fix $\Delta^{+}(\mathfrak{g}, \mathfrak{h})=\left\{e_{i}-e_{j}: i<j\right\}$ and $\left\{\alpha_{i}=e_{i}-e_{i+1}: 1 \leq i \leq n\right\}$ becomes a simple root system. Taking $\mathfrak{a}_{0}=i \mathbb{R}\left(E_{11}-E_{n+1 n+1}\right)$, then $\alpha_{1}$ and $\alpha_{n}$ have non-vanishing restriction to $\mathfrak{a}_{0}$ and $\beta_{0}=e_{1}-e_{n+1}$. In this case $\mathfrak{m} \simeq$ $\mathbb{C} \oplus \mathfrak{s l}(n-1, \mathbb{C})$. The center of $\mathfrak{m}$ is $\mathbb{C} H_{0}$, where

$$
H_{0}=\left(\begin{array}{ccc}
-(n-1) i & & \\
& 2 i I & \\
& & -(n-1) i
\end{array}\right)
$$

The fundamental weights of $\mathfrak{g}$ with respect to this simple root system are $\lambda_{i}=e_{1}+\cdots+e_{i}$, so it follows that $\lambda_{1}+\lambda_{n}=\beta_{0}$ and $\lambda_{1}-\lambda_{n}$ is the coordinate function of the center of $\mathfrak{m}$.

Then, if $\mu$ is strongly dominant integral, $\mu=m\left(\lambda_{1}-\lambda_{n}\right)+m_{2} \lambda_{2}^{M}+\cdots+$ $m_{n-1} \lambda_{n-1}^{M}$ with $m_{i} \in \mathbb{N}$ for all $2 \leq i \leq n-1, m \in \mathbb{R}$. It's easy to see that $\mu$ 
analytically integral implies $m \in \mathbb{Z}$. Then

$$
\begin{aligned}
k \beta_{0}+\mu & =k \beta_{0}+m\left(\lambda_{1}-\lambda_{n}\right)+\sum_{i=2}^{n-1} m_{i}\left(\lambda_{i}-k_{i} \beta_{0}\right) \\
& =\left(k+m-\sum_{i=2}^{n-1} m_{i} k_{i}\right) \lambda_{1}+\left(k-m-\sum_{i=2}^{n-1} m_{i} k_{i}\right) \lambda_{n}+\sum_{i=2}^{n-1} m_{i} \lambda_{i},
\end{aligned}
$$

and we choose $k=k_{0}+|m|+\sum_{i=2}^{n-1} m_{i} k_{i}$ with $k_{0} \in \mathbb{Z}^{+}$minimum subject to the condition $k \beta_{0}+\mu \notin F$.

Proof of Proposition 5.4. It suffices to take $\lambda=\mathbf{c}_{\beta}^{-1}\left(k \beta_{0}+\mu\right)$, with $k$ given by Lemma 5.7 , reminding that we are supposing $G^{\mathbb{C}}$ simply connected.

Proof of Proposition 5.3. By hypothesis there exists a constant $C$ and a positive integer $k$ such that

$$
\left|P_{\lambda, \tau}\right| \geq \frac{C}{\left(1+|\lambda|^{2}\right)^{k}\left(1+|\tau|^{2}\right)^{k}} \quad \forall(\lambda, \tau) \in\left(\mathcal{S}_{d}-F\right) \times \hat{K} .
$$

Besides, according to the choice of $\mathfrak{t}_{0}$ and $\mathfrak{h}_{0}, \gamma^{G}=\mathbf{c}_{\beta}^{-1} \circ \gamma_{\mathfrak{h}}^{G}$, therefore

$$
\begin{aligned}
P_{\lambda, \tau} & =\left(\left(\gamma^{G} \otimes \gamma^{K}\right)(P)\right)\left(\lambda, \mu_{\tau}\right) \\
& =\left(\left(\gamma_{\mathfrak{h}}^{G} \otimes \gamma^{K}\right)(P)\right)\left(\lambda \circ \mathbf{c}_{\beta}^{-1}, \mu_{\tau}\right) \\
& =\left(\left(\gamma_{\mathfrak{h}}^{G} \otimes \gamma^{K}\right)(P)\right)\left(\mathbf{c}_{\beta}(\lambda), \mu_{\tau}\right) ;
\end{aligned}
$$

given $\sigma \in \hat{M}$, let $\lambda \in \mathcal{S}_{d}-F$ be given by Proposition 5.4. If we set $x_{\sigma}=$ $\left.\mathbf{c}_{\beta}(\lambda)\right|_{\mathfrak{a}_{0}} \in i \mathfrak{a}_{0}^{\prime}$, then

$$
\begin{aligned}
P_{\sigma, \tau}\left(x_{\sigma}\right) & =\left(\left(\gamma_{\mathfrak{h}}^{G} \otimes \gamma^{K}\right)(P)\right)\left(x_{\sigma}+\mu_{\sigma}, \mu_{\tau}\right) \\
& =\left(\left(\gamma_{\mathfrak{h}}^{G} \otimes \gamma^{K}\right)(P)\right)\left(\mathbf{c}_{\beta}(\lambda), \mu_{\tau}\right)=P_{\lambda, \tau} .
\end{aligned}
$$

If $P$ has order $m$ in $\mathcal{U}(\mathfrak{g}), P_{\sigma, \tau}$ is a polynomial function on $\mathfrak{a}^{\prime}$ of order $\leq m$, and $\left\|P_{\sigma, \tau}\right\|$ is the norm of the vector in $\mathbb{C}^{m+1}$ formed with its coefficients, and by Schwarz inequality

$$
\left|P_{\sigma, \tau}\left(x_{\sigma}\right)\right| \leq\left\|P_{\sigma, \tau}\right\|\left(\sum_{j=0}^{m}\left|x_{\sigma}\right|^{2 m}\right)^{1 / 2} \leq\left\|P_{\sigma, \tau}\right\|\left(1+\left|x_{\sigma}\right|^{2}\right)^{\frac{m}{2}}
$$

besides,

$$
1+\left|x_{\sigma}\right|^{2} \leq C_{2}\left(1+\left|\mu_{\sigma}\right|^{2}\right)
$$

SO

$$
\left\|P_{\sigma, \tau}\right\| \geq \frac{\tilde{C}}{\left(1+|\sigma|^{2}\right)^{k+\frac{m}{2}}\left(1+|\tau|^{2}\right)^{k}} \quad \forall(\sigma, \tau) \in \hat{M} \times \hat{K},
$$

and it's enough to choose $\tilde{k}=k+\frac{m}{2}$. 


\section{Inversion of global characters.}

One important step in building the fundamental solution of $P$ is the construction of distributions $R_{\pi}$ such that $P R_{\pi}=\Theta_{\pi}$ for each representation $\pi$ that appears in the Plancherel formula. In this section we will define these distributions $R_{\pi}$. We will begin with the principal series representations. Before this we state some necessary results in order to bound the $R_{\pi}$. The next two lemmas follow directly from [14, Lemma 5.6.4].

Lemma 6.1. Let $K$ be a connected compact Lie group. There exist $\Omega \in$ $\mathcal{Z}(\mathfrak{k})$ and a positive constant $C$ such that

$$
\left|\chi_{\tau}(\Omega)\right| \geq C\left(1+|\tau|^{2}\right) \quad \forall \tau \in \hat{K} .
$$

Lemma 6.2. Let $G$ a connected semisimple Lie group with a compact Cartan subgroup. There exist $Z \in \mathcal{Z}(\mathfrak{g})$ and a positive constant $C$ such that

$$
\left|\chi_{\lambda}(Z)\right| \geq C\left(1+|\lambda|^{2}\right) \quad \forall \lambda \in \mathcal{S}_{d} .
$$

Proposition 6.3. Let $G$ a connected semisimple Lie group. There exist $Z \in \mathcal{Z}(\mathfrak{g})$, a positive constant $C$, an $\varepsilon>0$ and a positive integer $k$ such that

$$
\left|\chi_{\sigma, \nu+z}(Z)\right| \geq C\left(1+|\sigma|^{2}\right)^{k}\left(1+|\nu|^{2}\right)^{k} \quad \forall \sigma \in \hat{M}, \nu \in i \mathfrak{a}_{0}^{\prime}, z \in \mathfrak{a}^{\prime},|z|<\varepsilon .
$$

To prove this proposition, we begin with a lemma.

Lemma 6.4. Let $\mathfrak{g}_{0}$ be the Lie algebra of a semisimple Lie group $G, \mathfrak{h}_{0}$ a Cartan subalgebra of $\mathfrak{g}_{0}$, W the Weyl group of $\mathfrak{g}$. There exists $P \in \mathcal{S}(\mathfrak{h})^{W}$ an homogeneous polynomial function on $\mathfrak{h}^{\prime}$ such that

$$
P(\lambda)>0 \quad \forall \lambda \in i \mathfrak{h}_{0}^{\prime}, \lambda \neq 0 .
$$

Proof. We will begin with the construction of a $G$-invariant polynomial function on $\mathfrak{g}_{0}$. The symmetric algebra $\mathcal{S}\left(\mathfrak{g}_{0}\right)$ (resp. $\left.\mathcal{S}\left(\mathfrak{g}_{0}^{\prime}\right)\right)$ is identified with the set of polynomial functions on $\mathfrak{g}_{0}^{\prime}$ (resp. $\left.\mathfrak{g}_{0}\right)$. We denote $I\left(\mathfrak{g}_{0}\right)$ (resp. $I\left(\mathfrak{g}_{0}^{\prime}\right)$ ) the $G$-invariant elements of $\mathcal{S}\left(\mathfrak{g}_{0}\right)$ (resp. $\left.\mathcal{S}\left(\mathfrak{g}_{0}^{\prime}\right)\right)$.

The Killing form $B$ of $\mathfrak{g}_{0}$, being non-degenerate and $G$-invariant, induces canonical isomorphisms between $\mathcal{S}\left(\mathfrak{g}_{0}\right)$ and $\mathcal{S}\left(\mathfrak{g}_{0}^{\prime}\right)$ and between $I\left(\mathfrak{g}_{0}\right)$ and $I\left(\mathfrak{g}_{0}^{\prime}\right)$.

Given $X \in \mathfrak{g}_{0}$, let

$$
p_{X}(x)=\operatorname{det}(x I-\operatorname{ad} X)=x^{n}+a_{n-1}(X) x^{n-1}+\cdots+a_{l}(X) x^{l}
$$

be the characteristic polynomial of ad $X, n=\operatorname{dim} \mathfrak{g}_{0}, l=\operatorname{dim} \mathfrak{h}_{0}$. The coefficients $a_{i}(X)$ are $G$-invariant homogeneous polynomial functions of degree $n-i$ on $\mathfrak{g}_{0}$. Let $d_{i}$ and $p$ be positive integers such that $n-i+d_{i}=4 p$; if we define

$$
\tilde{Q}=a_{l}^{d_{l}}+\cdots+a_{n-1}^{d_{n-1}},
$$

then $\tilde{Q} \in I\left(\mathfrak{g}_{0}^{\prime}\right)$ and is homogeneous of degree $4 p$. 
$B$ is non-degenerate on $\mathfrak{h}_{0}$, so $\mathfrak{g}_{0}=\mathfrak{h}_{0} \oplus \mathfrak{h}_{0}^{\perp}, \mathfrak{h}_{0}^{\perp}$ the orthogonal of $\mathfrak{h}_{0}$ with respect to $B$. Besides $\mathfrak{h}_{0}^{\perp}=\left(\mathfrak{g}^{+} \oplus \mathfrak{g}^{-}\right) \cap \mathfrak{g}_{0}$ so $\tilde{Q}$ vanishes on $\mathfrak{h}_{0}^{\perp}$ and is positive on $\mathfrak{h}_{0}$. Let $Q \in I\left(\mathfrak{g}_{0}\right)$ be the image of $\tilde{Q}$ by the isomorphism mentioned above.

We also note that we can include $\mathfrak{h}_{0}^{\prime}$ in $\mathfrak{g}_{0}^{\prime}$ by extending a functional from $\mathfrak{h}_{0}$ to $\mathfrak{g}_{0}$ by 0 on $\mathfrak{h}_{0}^{\perp}$. This inclusion allow us to restrict elements from $\mathcal{S}\left(\mathfrak{g}_{0}\right)$ to $\mathcal{S}\left(\mathfrak{h}_{0}\right)$.

Let $P \in \mathcal{S}\left(\mathfrak{h}_{0}\right)$ be the restriction of $Q . P$ has the following properties:

(i) $P(\lambda)>0$ for all $\lambda \in \mathfrak{h}_{0}^{\prime}, \lambda \neq 0$.

(ii) $P \in \mathcal{S}(\mathfrak{h})^{W}$.

It only remains to see (ii): It's clear that $I\left(\mathfrak{g}_{0}\right)$ is included in $I(\mathfrak{g})$. Besides, $Q=P+\tilde{P}$, with $\tilde{P} \in \mathcal{S}\left(\mathfrak{g}_{0}\right) \mathfrak{h}_{0}^{\perp} \subset \mathcal{S}(\mathfrak{g})\left(\mathfrak{g}^{+} \oplus \mathfrak{g}^{-}\right)$. Now, $W_{G}=W\left(G_{u}, H_{u}\right)$ ([10, Thm. 4.41]), so, if $w \in W_{G}, w=\operatorname{Ad}(x)$, with $x \in N_{G_{u}}\left(H_{u}\right)$, and the action of $w$ preserves $\mathfrak{h}$ and $\mathfrak{g}^{+} \oplus \mathfrak{g}^{-}$, then $Q=w Q=w P+w \tilde{P}$, therefore $w P=P, w \tilde{P}=\tilde{P}$.

Finally, if $\lambda \in i \mathfrak{h}_{0}^{\prime}, P(\lambda)=(-i)^{4 p} P(i \lambda)>0$.

Proof of Proposition 6.3. Let $P \in \mathcal{S}(\mathfrak{h})^{W}$ be given by the lemma above. If

$$
2 c=\inf \left\{P(\lambda): \lambda \in i \mathfrak{h}_{0}^{\prime},|\lambda|=1\right\}>0,
$$

there exists $0<\varepsilon<1 / 2$ such that $\operatorname{Re} P(\lambda+\tilde{\lambda}) \geq c$ for all $\lambda \in i \mathfrak{h}_{0}^{\prime},|\lambda|=1$, $\tilde{\lambda} \in \mathfrak{h}^{\prime},|\tilde{\lambda}|<2 \varepsilon$. Suppose now that $\lambda \in i \mathfrak{h}_{0}^{\prime},|\lambda| \geq 1, \tilde{\lambda} \in \mathfrak{h}^{\prime},|\tilde{\lambda}|<\varepsilon$, then, as $\operatorname{Re} P$ is also an homogeneous polynomial of degree $4 p$, we have

$$
\operatorname{Re} P(\lambda+\tilde{\lambda})=|\lambda|^{4 p} \operatorname{Re} P\left(\frac{\lambda}{|\lambda|}+\frac{\tilde{\lambda}}{|\lambda|}\right) \geq|\lambda|^{4 p} c .
$$

On the other hand, if $|\lambda| \leq 1,|\tilde{\lambda}|<\varepsilon,|P(\lambda+\tilde{\lambda})|$ is bounded by a positive constant $A$. Now let $Z \in \mathcal{Z}(\mathfrak{g})$ be such that $\gamma_{\mathfrak{h}}(Z)=P+2 A$. If $\sigma \in \hat{M}$, $\nu \in i \mathfrak{a}_{0}^{\prime}, z \in \mathfrak{a}^{\prime},|z|<\varepsilon$, then, if $\left|\mu_{\sigma}+\nu\right| \leq 1$,

$$
\left|\chi_{\sigma, \nu+z}(P)\right| \geq A \geq C_{1}\left(1+\left|\mu_{\sigma}\right|^{2}\right)^{2 p}\left(1+|\nu|^{2}\right)^{2 p},
$$

and, if $\left|\mu_{\sigma}+\nu\right| \geq 1$,

$$
\left|\chi_{\sigma, \nu+z}(P)\right| \geq 2 A+\operatorname{Re} P\left(\mu_{\sigma}+\nu+z\right) \geq C_{2}\left(1+\left|\mu_{\sigma}\right|^{2}\right)^{2 p}\left(1+|\nu|^{2}\right)^{2 p} .
$$

Now we state a lemma we will use to obtain uniform bounds of the distributions $\Theta_{\sigma, \nu}$ and $\Theta_{\lambda}$. The proof follows easily from the proof of Theorem 10.2 in $[\mathbf{1 0}]$.

Lemma 6.5. Given $\tilde{K} \subset G$ a compact subset, for every admissible representation $\pi$ whose $K$-types have the multiplicity property $n_{\tau} \leq N \operatorname{dim} \tau$ there 
exists a differential operator $\tilde{\Omega} \in \mathcal{Z}(\mathfrak{k})$ and a constant $C$ which only depends on $\tilde{K}$ such that if supp $f \subseteq \tilde{K}$

$$
\left|\Theta_{\pi}(f)\right| \leq C N\left(\int_{G}|\tilde{\Omega} f(g)|^{2}\|\pi(g)\|^{2} d g\right)^{1 / 2} .
$$

In particular, if $\pi$ is unitary, $\left|\Theta_{\pi}(f)\right| \leq C N\|\tilde{\Omega} f\|_{L^{2}(G)}$.

6.1. Principal series. Before proceeding to the definition of the distributions $R_{\sigma, \nu}$ we state two lemmas about polynomial functions.

If $m$ is a positive integer we denote $\operatorname{Pol}(m)$ the complex vector space of polynomial functions in $\mathbb{C}^{n}$ of degree $\leq m$, and $\mathrm{Pol}^{0}(m)$ this vector space with the origin removed.

Given $Q \in \operatorname{Pol}(m), \zeta \in \mathbb{C}^{n}$, the equation

$$
\tilde{Q}(\zeta)=\left(\sum_{\alpha} \frac{1}{(\alpha !)^{2}}\left|Q^{(\alpha)}(\zeta)\right|^{2}\right)^{\frac{1}{2}}
$$

defines a norm in $\operatorname{Pol}(m)$ for a fixed $\zeta \in \mathbb{C}^{n}$. Let

$$
\|Q\|=\tilde{Q}(0) .
$$

The following lemma is used to construct a fundamental solution of a constant coefficients differential operator in $\mathbb{R}^{n}$ (Theorem 7.3 .10 of $[8]$ ). For a proof see Lemmas 7.3.11 and 7.3.12 in [8].

Lemma 6.6. For all $\varepsilon>0$ exists a non-negative function $\Phi \in C^{\infty}\left(\mathrm{Pol}^{0}(m)\right.$ $\left.\times \mathbb{C}^{n}\right)$ such that

(i) $\Phi$ is absolutely homogeneous of degree 0 , i.e., $\Phi(z Q, \zeta)=\Phi(Q, \zeta)$ for all $z \in \mathbb{C}-\{0\}$.

(ii) $\Phi(Q, \zeta)=0$ if $|\zeta| \geq \varepsilon$.

(iii) If $F$ is an entire function in $\mathbb{C}^{n}$ and $d \zeta$ is Lebesgue measure in $\mathbb{C}^{n}$,

$$
\int F(\zeta) \Phi(Q, \zeta) d \zeta=F(0)
$$

(iv) There exists a constant $C_{\varepsilon}$ such that $\tilde{Q}(0) \leq C_{\varepsilon}|Q(\zeta)|$ if $\Phi(Q, \zeta) \neq 0$.

$\operatorname{Being} \operatorname{Pol}(m)$ a finite dimensional vector space, all norms $\tilde{Q}(\zeta)$ are equivalent. The following lemma estimates the constants which give this equivalence. The proof is an easy consequence of Taylor's formula.

Lemma 6.7. Exists a constant $C>0$ depending only on $n$ and $m$ such that

$$
C\left(1+|\zeta|^{2}\right)^{-m} \tilde{Q}(0)^{2} \leq \tilde{Q}(\zeta)^{2} \leq C\left(1+|\zeta|^{2}\right)^{m} \tilde{Q}(0)^{2}
$$

for all $Q \in \operatorname{Pol}(m)$. 
We are now in position to define the distributions $R_{\sigma, \nu}$ for $P \in \mathcal{Z}\left(\mathcal{U}(\mathfrak{g})^{K}\right)$ satisfying the hypothesis of Theorem 1.1. Let $\varepsilon>0$ be given by Proposition $6.3, m$ the order of $P$ and let $\Phi \in C^{\infty}\left(\operatorname{Pol}^{0}(m) \times \mathbb{C}\right)$ be the non-negative function given by Lemma 6.6.

Given $\sigma \in \hat{M}, \tau \in \hat{K}$, and a fixed $\nu \in \mathfrak{a}^{\prime}$, we put

$$
P_{\sigma, \tau}^{\nu}(z)=P_{\sigma, \tau}(\nu+z)=\left(\chi_{\sigma, \nu+z} \otimes \chi_{\tau}\right)(P) .
$$

Finally, if $d z$ is Lebesgue measure in $\mathfrak{a}^{\prime}, f \in \mathcal{D}(G)$, we define

$$
R_{\sigma, \nu}(f)=\sum_{\tau \in \hat{K}} \int_{|z|<\varepsilon} \frac{1}{P_{\sigma, \tau}^{\nu}(z)} \Theta_{\sigma, \nu+z}^{\tau}(f) \Phi\left(P_{\sigma, \tau}^{\nu}, z\right) d z .
$$

This definition makes sense because $P_{\sigma, \tau}^{\nu}(z) \neq 0$ if $\Phi\left(P_{\sigma, \tau}^{\nu}, z\right) \neq 0$ (Lemma 6.6 (iv)).

Proposition 6.8. The map defined by (23) is a finite order distribution for all $\sigma \in \hat{M}, \nu \in \mathfrak{a}^{\prime}$. This map has also the following properties:

(i) $P R_{\sigma, \nu}=\Theta_{\sigma, \nu}$.

(ii) For every positive integer $k$ and $f \in \mathcal{D}(G)$, exist a constant $C>0$ which only depends on the support of $f$ and a differential operator $D_{k} \in \mathcal{Z}\left(\mathcal{U}(\mathfrak{g})^{K}\right)$ such that

$$
\left|R_{\sigma, \nu}(f)\right| \leq \frac{C}{\left(1+|\nu|^{2}\right)^{k}\left(1+|\sigma|^{2}\right)^{k}}\left\|D_{k} f\right\|_{L^{2}(G)} \quad \forall \sigma \in \hat{M}, \nu \in i \mathfrak{a}_{0}^{\prime} .
$$

Proof. By Proposition 3.2, $P \Theta_{\sigma, \nu+z}^{\tau}=P_{\sigma, \tau}^{\nu}(z) \Theta_{\sigma, \nu+z}^{\tau}$, and, as $\Theta_{\sigma, \nu+z}(f)$ is an entire function in $z$ ([6], Section 21) for fixed $f$, so it is $\Theta_{\sigma, \nu+z}^{\tau}(f)$, and by Lemma 6.6 (ii),

$$
\int_{|z|<\varepsilon} \Theta_{\sigma, \nu+z}^{\tau}(f) \Phi\left(P_{\sigma, \tau}^{\nu}, z\right) d z=\Theta_{\sigma, \nu}^{\tau}(f),
$$

and this proves (i).

Let's see that (23) defines a distribution: According to Lemma 6.6 (iv) and Lemma 6.7 together with (20) and (21), if $\Phi\left(P_{\sigma, \tau}^{\nu}, z\right) \neq 0$, we have ||$P_{\sigma, \tau} \| \leq C_{1}\left(1+|\nu|^{2}\right)^{m}\left|P_{\sigma, \tau}^{\nu}(z)\right|$, and by the hypothesis on $P$ (Prop. 5.1 or $5.3)$, exists a positive integer $\tilde{k}$ such that

$$
\frac{1}{\left|P_{\sigma, \tau}^{\nu}(z)\right|} \leq C_{2}\left(1+|\nu|^{2}\right)^{m}\left(1+|\sigma|^{2}\right)^{\tilde{k}}\left(1+|\tau|^{2}\right)^{\tilde{k}} .
$$

On the other hand, if $Z \in \mathcal{Z}(\mathfrak{g})$ and $\Omega \in \mathcal{Z}(\mathfrak{k})$ are given by Proposition 6.3 and Lemma 6.1 respectively and if $s_{1}$ and $s_{2}$ are positive integers, we have, 
for some positive integer $\bar{k}$,

$$
\begin{aligned}
\left|\Theta_{\sigma, \nu+z}^{\tau}(f)\right| & =\frac{\left|\Theta_{\sigma, \nu+z}^{\tau}\left(\left(Z^{t}\right)^{s_{1}}\left(\Omega^{t}\right)^{s_{2}} f\right)\right|}{\left|\chi_{\sigma, \nu+z}^{s_{1}}(Z)\right|\left|\chi_{\tau}^{s_{2}}(\Omega)\right|} \\
& \leq \frac{C_{3}\left|\Theta_{\sigma, \nu+z}\left(\left(Z^{t}\right)^{s_{1}}\left(\Omega^{t}\right)^{s_{2}} f_{\bar{\tau}}\right)\right|}{\left(\left(1+|\sigma|^{2}\right)\left(1+|\nu|^{2}\right)\right)^{s_{1}-\bar{k}}\left(1+|\tau|^{2}\right)^{s_{2}}},
\end{aligned}
$$

besides, it holds for $D \in \mathcal{U}(\mathfrak{g})^{K}$ that $D f_{\bar{\tau}}=(D f)_{\bar{\tau}}$; also $\left|\Theta_{\pi}\left(f_{\bar{\tau}}\right)\right| \leq\left|\Theta_{\pi}(f)\right|$, so

$$
\left|\Theta_{\sigma, \nu+z}^{\tau}(f)\right| \leq \frac{C_{3}\left|\Theta_{\sigma, \nu+z}\left(\left(Z^{t}\right)^{s_{1}}\left(\Omega^{t}\right)^{s_{2}} f\right)\right|}{\left(\left(1+|\sigma|^{2}\right)\left(1+|\nu|^{2}\right)\right)^{s_{1}-\bar{k}}\left(1+|\tau|^{2}\right)^{s_{2}}} .
$$

Let $\tilde{K} \subseteq G$ be a compact subset. Note that for principal series it holds $n_{\tau} \leq \operatorname{dim} \tau([\mathbf{1 0}$, p. 207]); so by Lemma 6.5 exist $\tilde{\Omega} \in \mathcal{Z}(\mathfrak{k})$ and a constant $C_{1}$ independent of $\sigma \in \hat{M}, \nu, z \in \mathfrak{a}^{\prime}$ such that

$$
\left|\Theta_{\sigma, \nu+z}(f)\right| \leq C_{1}\left(\int_{G}|\tilde{\Omega} f(g)|^{2}|| \pi_{\sigma, \nu+z}(g) \|^{2} d g\right)^{1 / 2} ;
$$

on the other hand, given $\varphi$ in the space $V^{\sigma}$ where the $\pi_{\sigma, \nu+z}$ acts, if $a(g)$ is the $A$-component of $g$ in the Iwasawa decomposition, then (cf. [10, p. 169]),

$$
\left(\pi_{\sigma, \nu+z}(g) \varphi\right)(k)=e^{-z \log a\left(g^{-1} k\right)}\left(\pi_{\sigma, \nu}(g) \varphi\right)(k),
$$

and taking $A=\sup _{g \in \tilde{K}, k \in K,|z|<\varepsilon}\left|e^{-z \log a\left(g^{-1} k\right)}\right|$ and $B_{\sigma, \nu}=\sup _{g \in \tilde{K}}\left\|\pi_{\sigma, \nu}(g)\right\|$, then $\left\|\pi_{\sigma, \nu+z}(g)\right\| \leq A B_{\sigma, \nu}$ uniformly on $\tilde{K}$, so for all $f$ such that supp $f \subseteq$ $\tilde{K}$,

$$
\left|\Theta_{\sigma, \nu+z}^{\tau}(f)\right| \leq A B_{\sigma, \nu}|| \tilde{\Omega} f \|_{L^{2}(G)} .
$$

Now combining (24), (25) and (26) and the fact that $\Phi$ is uniformly bounded we obtain

$$
\begin{aligned}
& \left|\int_{|z|<\varepsilon} \frac{\Theta_{\sigma, \nu+z}^{\tau}(f) \Phi\left(P_{\sigma, \tau}^{\nu}, z\right)}{P_{\sigma, \tau}^{\nu}(z)} d z\right| \\
& \leq \frac{C_{4} B_{\sigma, \nu}|| \tilde{\Omega}\left(Z^{t}\right)^{s_{1}}\left(\Omega^{t}\right)^{s_{2}} f \|_{L^{2}(G)}}{\left(1+|\nu|^{2}\right)^{s_{1}-m-\bar{k}}\left(1+|\sigma|^{2}\right)^{s_{1}-\tilde{k}-\bar{k}}\left(1+|\tau|^{2}\right)^{s_{2}-\tilde{k}}}
\end{aligned}
$$

therefore for all $f$ such that supp $f \subseteq \tilde{K}$

$$
\left|R_{\sigma, \nu}(f)\right| \leq\left(\sum_{\tau \in \hat{K}} \frac{1}{\left(1+|\tau|^{2}\right)^{s_{2}-\tilde{k}}}\right) \frac{C_{4} B_{\sigma, \nu}|| \tilde{\Omega}\left(Z^{t}\right)^{s_{1}}\left(\Omega^{t}\right)^{s_{2}} f \|_{L^{2}(G)}}{\left(1+|\nu|^{2}\right)^{s_{1}-m-\bar{k}}\left(1+|\sigma|^{2}\right)^{s_{1}-\tilde{k}-\bar{k}}}
$$

and $\sum_{\tau \in \hat{K}} \frac{1}{\left(1+|\tau|^{2}\right)^{s_{2}-\tilde{k}}}$ is finite if we choose $s_{2}>\tilde{k}+1 / 2 \operatorname{dim} K([\mathbf{1 4}$, Lemma 5.6.7]), so $R_{\sigma, \nu}$ is a finite order distribution. 
To see (ii), just observe that $B_{\sigma, \nu}=1$ if $\nu \in i \mathfrak{a}_{0}^{\prime}$, so given $k$ if we take $D_{k}=\tilde{\Omega}\left(Z^{t}\right)^{s_{1}}\left(\Omega^{t}\right)^{s_{2}}$ with the $s_{2}$ chosen above and $s_{1} \geq k+\bar{k}+\max (\tilde{k}, m)$, (27) becomes

$$
\left|R_{\sigma, \nu}(f)\right| \leq \frac{C}{\left(1+|\nu|^{2}\right)^{k}\left(1+|\sigma|^{2}\right)^{k}}\left\|D_{k} f\right\|_{L^{2}(G)}
$$

with $C$ depending only $\tilde{K}$.

6.2. Discrete Series. Suppose $T$ is a compact Cartan subgroup of $G$.

Proposition 6.9. Let $P \in \mathcal{Z}\left(\mathcal{U}(\mathfrak{g})^{K}\right)$ such that exist a constant $C$ and a positive integer $k$ such that

$$
\left|P_{\lambda, \tau}\right| \geq \frac{C}{\left(1+|\lambda|^{2}\right)^{k}\left(1+|\tau|^{2}\right)^{k}} \quad \forall(\lambda, \tau) \in \mathcal{S}_{d} \times \hat{K},
$$

then the map

$$
R_{\lambda}(f)=\sum_{\tau \in \hat{K}} \frac{1}{P_{\lambda, \tau}} \Theta_{\lambda}^{\tau}(f)
$$

defines a finite order distribution with the following properties:

(i) $P R_{\lambda}=\Theta_{\lambda}$.

(ii) For each positive integer $k$ and $f \in \mathcal{D}(G)$, exist a constant $C>0$ which only depends on the support of $f$ and a differential operator $E_{k} \in \mathcal{Z}\left(\mathcal{U}(\mathfrak{g})^{K}\right)$ such that

$$
\left|R_{\lambda}(f)\right| \leq \frac{C}{\left(1+|\lambda|^{2}\right)^{k}}\left\|E_{k} f\right\|_{L^{2}(G)} \quad \forall \lambda \in \mathcal{S}_{d}
$$

Proof. First note that $R_{\lambda}$ is well defined because by Proposition 5.2 exist a constant $C_{1}$ and a positive integer $k_{1}$ such that

$$
\left|P_{\lambda, \tau}\right| \geq \frac{C_{1}}{\left(1+|\lambda|^{2}\right)_{1}^{k}\left(1+|\tau|^{2}\right)_{1}^{k}} \quad \forall(\lambda, \tau) \in \mathcal{S}_{d} \times \hat{K},
$$

and in particular $P_{\lambda, \tau} \neq 0$; (i) is clear because $P \Theta_{\lambda}^{\tau}=P_{\lambda, \tau} \Theta_{\lambda}^{\tau}$ (Proposition $3.2)$.

To see (ii), observe that by Lemma 6.5 exist a constant $C_{2}$ depending only on $\operatorname{supp} f$ and $\tilde{\Omega} \in \mathcal{Z}(\mathfrak{k})$ such that $\left|\Theta_{\lambda}(f)\right| \leq C_{2}\|\tilde{\Omega} f\|_{L^{2}(G)}$. 
Then, if $Z \in \mathcal{Z}(\mathfrak{g})$ and $\Omega \in \mathcal{Z}(\mathfrak{k})$ are given by Lemmas 6.2 and 6.1 respectively, we have

$$
\begin{aligned}
\left|R_{\lambda}(f)\right| & \leq \sum_{\tau \in \hat{K}} \frac{1}{\left|P_{\lambda, \tau}\right|}\left|\Theta_{\lambda}^{\tau}(f)\right| \leq \sum_{\tau \in \hat{K}} \frac{1}{C_{3}}\left(1+|\lambda|^{2}\right)_{1}^{k}\left(1+|\tau|^{2}\right)_{1}^{k}\left|\Theta_{\lambda}\left(f_{\bar{\tau}}\right)\right| \\
& \leq \sum_{\tau \in \hat{K}} \frac{\tilde{C}}{\left(1+|\lambda|^{2}\right)^{s_{1}-k_{1}}\left(1+|\tau|^{2}\right)^{s_{2}-k_{1}}}\left|\Theta_{\lambda}\left(\left(Z^{t}\right)^{s_{1}}\left(\Omega^{t}\right)^{s_{2}} f_{\bar{\tau}}\right)\right| \\
& \leq\left(\sum_{\tau \in \hat{K}} \frac{1}{\left(1+|\tau|^{2}\right)^{s_{2}-k_{1}}}\right) \frac{\tilde{C} C_{1}}{\left(1+|\lambda|^{2}\right)^{s_{1}-k_{1}}}\left\|\left(\tilde{\Omega}\left(Z^{t}\right)^{s_{1}}\left(\Omega^{t}\right)^{s_{2}} f\right)\right\|_{L^{2}(G)},
\end{aligned}
$$

and it suffices to take $E_{k}=\tilde{\Omega}\left(Z^{t}\right)^{s_{1}}\left(\Omega^{t}\right)^{s_{2}}$ with $s_{1}=k+k_{1}$ and $s_{2}$ such that $\sum_{\tau \in \hat{K}} \frac{1}{\left(1+|\tau|^{2}\right)^{s_{2}-k_{1}}}$ is finite.

\section{Demonstration of Theorem 1.1.}

Now we are ready to complete the proof of Theorem 1.1 with the explicit construction of the fundamental solution of $P$.

Proposition 7.1. Let $P \in \mathcal{Z}\left(\mathcal{U}(\mathfrak{g})^{K}\right)$ and suppose that exist a constant $C$ and a positive integer $k$ such that in case $\mathrm{I}$,

$$
\left\|P_{\sigma, \tau}\right\| \geq \frac{C}{\left(1+|\sigma|^{2}\right)^{k}\left(1+|\tau|^{2}\right)^{k}} \quad \forall(\sigma, \tau) \in \hat{M} \times \hat{K},
$$

and in case II,

$$
\left|P_{\lambda, \tau}\right| \geq \frac{C}{\left(1+|\lambda|^{2}\right)^{k}\left(1+|\tau|^{2}\right)^{k}} \quad \forall(\lambda, \tau) \in \mathcal{S}_{d} \times \hat{K} ;
$$

if $R_{\sigma, \nu}$ and $R_{\lambda}$ are the distributions defined respectively by (23) and 6.9, then the map $R$ defined in case I,

$$
R=\sum_{\sigma \in \hat{M}} \int_{\nu \in i \mathfrak{a}_{0}^{\prime}} R_{\sigma, \nu} m_{\sigma}(\nu) d \nu
$$

and in case II,

$$
R=\sum_{\sigma \in \hat{M}} \int_{\nu \in i \mathfrak{a}_{0}^{\prime}} R_{\sigma, \nu} m_{\sigma}(\nu) d \nu+\sum_{\lambda \in \mathcal{S}_{d}} d_{\lambda} R_{\lambda},
$$

is a finite order distribution which is a fundamental solution of $P$.

Remark. We note that in case II, $T$ is a fundamental Cartan subgroup of $G$, and in case I, $H=A \times T^{-}$is. 
So if $P \in \mathcal{Z}\left(\mathcal{U}(\mathfrak{g})^{K}\right)$ is such that $\left(\gamma^{G} \otimes \gamma^{K}\right)(P)$ has a fundamental solution in $H^{f} \times T$, Propositions 5.2 and 5.1 imply (28) and (29) respectively in each case, so Theorem 1.1 is a direct consequence of Proposition 7.1.

Proof. First of all, we note that in case II, Proposition 5.3 says that (28) implies (29) (changing, maybe, $C$ and $k$ ), so $R_{\sigma, \nu}$ is well defined and Proposition 6.8 applies in this case.

Equality $P R=\delta$ is clear by Plancherel formula (Theorem 2.1) and because $P R_{\sigma, \nu}=\Theta_{\sigma, \nu}$ and $P R_{\lambda}=\Theta_{\lambda}$ (Propositions 6.8 and 6.9 respectively); it only remains to prove that $R$ is a finite order distribution in each case. So we will prove that each of the following are finite order distributions:

$$
R_{s p}=\sum_{\sigma \in \hat{M}} \int_{\nu \in i \mathfrak{a}_{0}^{\prime}} R_{\sigma, \nu} m_{\sigma}(\nu) d \nu, \quad R_{s d}=\sum_{\lambda \in \mathcal{S}_{d}} d_{\lambda} R_{\lambda}
$$

Let $\tilde{K}$ be a compact subset and $f \in \mathcal{D}_{\tilde{K}}(G)$; for each positive integer $k$ let $D_{k} \in \mathcal{Z}\left(\mathcal{U}(\mathfrak{g})^{K}\right)$ be given by Proposition 6.8 (ii), then, using 2.1 (ii),

$$
\left|R_{s p}(f)\right| \leq C_{1} C_{2}\left(\sum_{\sigma \in \hat{M}} \frac{1}{\left(1+|\sigma|^{2}\right)^{k-l_{2}}}\right)\left(\int_{\nu \in i \mathfrak{a}_{0}^{\prime}} \frac{1}{\left(1+|\nu|^{2}\right)^{k-l_{1}}}\right)\left\|D_{k} f\right\|_{L^{2}(G)},
$$

and choosing $k$ large enough so that the sum and the integral are finite, we obtain an operator $D \in \mathcal{Z}\left(\mathcal{U}(\mathfrak{g})^{K}\right)$ and a constant $C$ depending only on $\tilde{K}$ such that

$$
\left|R_{s p}(f)\right| \leq C|| D f \|_{L^{2}(G)},
$$

and this proves that $R_{s p}$ is a distribution of finite order less or equal that the order of $D$.

In the same way, for each positive integer $k$ let $E_{k} \in \mathcal{Z}\left(\mathcal{U}(\mathfrak{g})^{K}\right)$ be given by Proposition 6.9 (ii), therefore

$$
\left|R_{s d}(f)\right| \leq C_{3}\left(\sum_{\tau \in \hat{K}} \frac{d_{\lambda}}{\left(1+|\lambda|^{2}\right)^{k}}\right)\left\|E_{k} f\right\|_{L^{2}(G)}
$$

if $k>1 / 2 \operatorname{dim} G, \sum_{\tau \in \hat{K}} \frac{d_{\lambda}}{\left(1+|\lambda|^{2}\right)^{k}}$ is finite $([\mathbf{1 4}$, Lemma 5.6.7]), there exist $E \in \mathcal{Z}\left(\mathcal{U}(\mathfrak{g})^{K}\right)$ and a constant $\tilde{C}$ depending only on $\tilde{K}$ such that

$$
\left|R_{s d}(f)\right| \leq \tilde{C}\|E f\|_{L^{2}(G)},
$$

so $R_{s d}$ is a distribution of finite order less or equal that the order of $E$. 


\section{P-convexity of $G$.}

Suppose that $P \in \mathcal{Z}\left(\mathcal{U}(\mathfrak{g})^{K}\right)$ satisfies the conditions of Proposition 7.1. So $P$ has a fundamental solution $R \in \mathcal{D}^{\prime}(G)$. This implies that the differential equation $P u=f$ has a solution $u \in C^{\infty}(G)$ for all $f \in \mathcal{D}(G)$; just taking $u=f * R$ because $P u=f * P R=f * \delta=f$. Now, in order to guarantee the solvability of $P u=f$ when $f \in C^{\infty}(G)$, it is necessary to analyze the $P$-convexity of $G$.

Definition 2. Given $D \in \mathcal{U}(\mathfrak{g})$, we say that $G$ is $D$-convex if for every compact subset $\Omega \subseteq G$ exists another compact subset $\tilde{\Omega} \subseteq G$ such that

$$
\operatorname{supp}(D f) \subseteq \Omega \Longrightarrow \operatorname{supp}(f) \subseteq \tilde{\Omega} .
$$

Using Johnson's injectivity criterion ([9]), we will verify that $G$ is $P$ convex.

Let $P \in \mathcal{Z}\left(\mathcal{U}(\mathfrak{g})^{K}\right)$ satisfying the conditions of 7.1. Then $P_{\sigma, \tau}$ is a non-zero polynomial on $\mathfrak{a}^{\prime}$ for all $(\sigma, \tau) \in \hat{M} \times \hat{K}$.

Given $\sigma \in \hat{M}, \nu \in \mathfrak{a}^{\prime}$, we denote $V^{\sigma}$ the space where the principal series representation $\pi_{\sigma, \nu}$ acts (remind that we can choose $V^{\sigma}$ independent of $\nu$ ). Let $V_{F}^{\sigma}$ be the subspace of $K$-finite vectors of $\pi_{\sigma, \nu}$.

Now, if $U$ is a $\pi_{\sigma, \nu}(P)$-invariant finite dimensional subspace of $V_{F}^{\sigma}$, then

$$
U \subseteq W=\sum_{j=i}^{k} n_{\tau_{j}}^{\sigma} V_{\tau_{j}}
$$

on the other hand, if $v_{j} \in V_{\tau_{j}}, \pi_{\sigma, \nu}(P) v_{j}=\left(\chi_{\sigma, \nu} \otimes \chi_{\tau_{j}}\right)(P) v_{j}=P_{\sigma, \tau_{j}}(\nu) v_{j}$, that is, $\pi_{\sigma, \nu}(P)$ is diagonalizable on $W$, so $U$ has a $\pi_{\sigma, \nu}(P)$-invariant complement $\tilde{U}$ in $W$.

Suppose that $\left.\operatorname{det} \pi_{\sigma, \nu}(P)\right|_{U}=0$ for all $\nu \in \mathfrak{a}^{\prime}$. Then

$$
0=\left.\left.\operatorname{det} \pi_{\sigma, \nu}(P)\right|_{U} \operatorname{det} \pi_{\sigma, \nu}(P)\right|_{\tilde{U}}=\prod_{j=1}^{k}\left(P_{\sigma, \tau_{j}}(\nu)\right)^{d_{\tau_{j}} n_{\tau_{j}}^{\sigma}} \quad \forall \nu \in \mathfrak{a}^{\prime},
$$

therefore exist $\sigma \in \hat{M}, \tau \in \hat{K}$ such that $P_{\sigma, \tau}(\nu)=0$ for all $\nu \in \mathfrak{a}^{\prime}$ which is absurd. So Theorems 5.1 and 5.2 in [9] imply that $G$ is $P$-convex.

\section{Parametrix of operators in $\mathcal{Z}\left(\mathcal{U}(\mathfrak{g})^{K}\right)$.}

In this section, using Zuckerman characters identities combined with the work done so far, we will prove:

Proposition 9.1. Let $G$ be a linear connected semisimple Lie group of rank one having a compact Cartan subgroup, and $P \in \mathcal{Z}\left(\mathcal{U}(\mathfrak{g})^{K}\right)$. A sufficient condition for the existence of a parametrix of $P$ is: 
Exist a finite set $F \subset \mathcal{S}_{d}$, a positive constant $C$ and a positive integer $k$ such that

$$
\left|P_{\lambda, \tau}\right| \geq \frac{C}{\left(1+|\lambda|^{2}\right)^{k}\left(1+|\tau|^{2}\right)^{k}} \quad \forall(\lambda, \tau) \in\left(\mathcal{S}_{d}-F\right) \times \hat{K} .
$$

Remark. The fact that if $\left(\gamma^{G} \otimes \gamma^{K}\right)(P)$ has a parametrix on $T \times T$ then (32) holds is an easy consequence of [4, Thm. II]. So Proposition 9.1 clearly implies Theorem 1.3.

Before proceeding to the proof of this proposition, we state Zuckerman characters identities in the form we need (cf. Proposition 5.13 from [16] for a more precise statement together with the proof).

Proposition 9.2. Let $G$ be as in Proposition 9.1. We put $r=\frac{|W(\mathfrak{g}, \mathfrak{h})|}{|W(G, H)|}$. Given $\lambda \in \mathcal{S}_{d}$ exist $\sigma_{1}^{\lambda}, \ldots, \sigma_{r}^{\lambda} \in \hat{M}$ and $\nu_{1}^{\lambda}, \ldots, \nu_{r}^{\lambda} \in \mathfrak{a}_{0}^{\prime}$ such that

$$
\frac{1}{\left|W_{K}\right|} \sum_{w \in W_{G}} \Theta_{w \lambda}=a_{0}(\lambda) \Theta_{\lambda}^{f}+\sum_{j=1}^{r} a_{j}(\lambda) \Theta_{\sigma_{j}^{\lambda}, \nu_{j}^{\lambda}},
$$

where $\Theta_{\lambda}^{f}$ is the character of the finite dimensional representation with infinitesimal character $\lambda$, and $a_{j}(\lambda)= \pm 1$ for all $0 \leq j \leq r, \lambda \in \mathcal{S}_{d}$.

Proof of Proposition 9.1. We will construct the parametrix $R$ of $P$ as the sum of three distributions $R=R_{s p}+R_{s d}+R_{F}$.

As in the proof of Proposition 7.1, applying Proposition 5.3 together with 6.8 , we see that

$$
R_{s p}=\sum_{\sigma \in \hat{M}} \int_{\nu \in i \mathfrak{a}_{0}^{\prime}} R_{\sigma, \nu} m_{\sigma}(\nu) d \nu
$$

defines a finite order distribution such that

$$
P R_{s p}=\sum_{\sigma \in \hat{M}} \int_{\nu \in i \mathfrak{a}_{0}^{\prime}} \Theta_{\sigma, \nu} m_{\sigma}(\nu) d \nu .
$$

In the same way, if we define

$$
R_{s d}=\sum_{\lambda \in \mathcal{S}_{d}-F} d_{\lambda} R_{\lambda}
$$

then, applying Proposition 6.9,

$$
P R_{s d}=\sum_{\lambda \in \mathcal{S}_{d}-F} d_{\lambda} \Theta_{\lambda} .
$$

For the definition of $R_{F}$, we note in first place that having all the representations $\pi_{w \lambda}$ infinitesimal character $\lambda, F$ is closed by the action of $W_{G}$. 
Besides $\pi_{w \lambda}$ is equivalent to $\pi_{w^{\prime} \lambda}$ if and only if $w^{-1} w^{\prime} \in W_{K}$, and we can write

$$
\sum_{\lambda \in F} \Theta_{\lambda}=\frac{\left|W_{K}\right|}{\left|W_{G}\right|} \sum_{\lambda \in F} \frac{1}{\left|W_{K}\right|} \sum_{w \in W_{G}} \Theta_{w \lambda}
$$

Now, for each $\lambda \in F$, let $\sigma_{1}^{\lambda}, \ldots, \sigma_{r}^{\lambda} \in \hat{M}$ and $\nu_{1}^{\lambda}, \ldots, \nu_{r}^{\lambda} \in \mathfrak{a}_{0}^{\prime}$ be given by Proposition 9.2; then we can define

$$
R_{F}=\frac{\left|W_{K}\right|}{\left|W_{G}\right|} \sum_{\lambda \in F}\left(\sum_{j=1}^{r} a_{j}(\lambda) R_{\sigma_{j}^{\lambda}, \nu_{j}^{\lambda}}\right)
$$

which is a finite order distribution.

Finally, Propositions 6.8 and 9.2 imply

$$
P R_{F}=\frac{\left|W_{K}\right|}{\left|W_{G}\right|} \sum_{\lambda \in F}\left(\sum_{j=1}^{r} a_{j}(\lambda) \Theta_{\sigma_{j}^{\lambda}, \nu_{j}^{\lambda}}\right)=\sum_{\lambda \in F} \Theta_{\lambda}-\frac{\left|W_{K}\right|}{\left|W_{G}\right|} \sum_{\lambda \in F} a_{o}(\lambda) \Theta_{\lambda}^{f} ;
$$

therefore, putting $R=R_{s p}+R_{s d}+R_{F}$, the above equalities together with Plancherel formula (Theorem 2.1) imply

$$
P R-\delta=-\frac{\left|W_{K}\right|}{\left|W_{G}\right|} \sum_{\lambda \in F} a_{0}(\lambda) \Theta_{\lambda}^{f},
$$

and this distribution is given by a $C^{\infty}$ function because $\Theta_{\lambda}^{f}$ are characters of a finite dimensional representation.

\section{Casimir Operator.}

The fundamental solution constructed for $P \in \mathcal{Z}\left(\mathcal{U}(\mathfrak{g})^{K}\right)$ satisfying the conditions of Theorem 1.1 is invariant by inner automorphisms of $K$. In the case that $P$ is a bi-invariant operator in the conditions of Corollary 1.2 , we obtain a fundamental solution of $P$ invariant by inner automorphisms of all $G$.

Let's analyze the case of the Casimir operator of $G$. If $\mathfrak{h}$ is a Cartan subalgebra of $\mathfrak{g}$, and if $\lambda \in \mathfrak{h}^{\prime}$, then $\chi_{\lambda}(\Omega)=B(\lambda, \lambda)-B(\rho, \rho)([\mathbf{1 4}, 5.6 .4])$; in particular, if $\sigma \in \hat{M}, \nu \in i \mathfrak{a}_{0}^{\prime}$,

$$
\chi_{\sigma, \nu}(\Omega)=\chi_{\mu_{\sigma}+\nu}(\Omega)=\left|\mu_{\sigma}\right|-|\nu|^{2}-|\rho|^{2},
$$

and $\left\|\chi_{\mu,-}(\Omega)\right\| \geq 1$ for all $\mu \in \widehat{T^{-}}$, so, by Theorem $4.1, \gamma_{\mathfrak{h}}^{G}(\Omega)$ has a fundamental solution on $A \times T^{-}$. Besides if $\mathfrak{t}$ is a Cartan subalgebra of $\mathfrak{g}$, in this case if $\lambda \in i \mathfrak{t}_{0}^{\prime}$ we have

$$
\chi_{\lambda}(\Omega)=|\lambda|^{2}-|\rho|^{2}
$$


now, being $G^{\mathbb{C}}$ simply connected, $\lambda=\rho$ is a discrete series parameter, and consequently we cannot apply Corollary 1.2.

So Theorem 1.1 only provides a fundamental solution of the Casimir operator when $G$ has one conjugacy class of Cartan subgroups.

We note, on the other hand, that solvability of $\Omega$ have been proved by Rauch and Wigner ([12]) in a non-constructive way. In [2] an explicit fundamental solution of $\Omega$ is constructed for $G=S L(2, \mathbb{R})$ and it's also proved that an invariant one doesn't exists for this group.

However, note that $\left|\chi_{\lambda}(\omega)\right| \geq C$ for all $\lambda \in \mathcal{S}_{d}-F$, where $F=\{\lambda \in \mathcal{S}$ : $|\lambda|=|\rho|\}$ is a finite subset, so by Proposition 9.1, $\Omega$ has a parametrix on $G$.

\section{A necessary condition.}

Let $G$ be a connected semisimple Lie group. Recall from [7, Thm. 5.17] that we can define a Harish-Chandra homomorphism $\gamma_{\mathfrak{a}}: \mathcal{U}(\mathfrak{g})^{K} \rightarrow \mathcal{S}(\mathfrak{a})^{W_{0}}$, where $W_{0}=W\left(\mathfrak{g}_{0}, \mathfrak{a}_{0}\right)$ is the Weyl group of the restricted root system. The kernel of $\gamma_{\mathfrak{a}}$ is $\mathcal{U}(\mathfrak{g})^{K} \cap \mathcal{U}(\mathfrak{g}) \mathfrak{k}$.

Proposition 4.1 in $[\mathbf{1 3}]$ states that if $P \in \mathcal{Z}(\mathfrak{g})$ is in the kernel of $\gamma_{\mathfrak{a}}$, then $P$ doesn't have a parametrix. In this section we will extend this proposition for $P \in \mathcal{Z}\left(\mathcal{U}(\mathfrak{g})^{K}\right)$.

Let $\mathcal{D}_{K}(G)$, resp. $C_{K}^{\infty}(G)$, be the space of left and right $K$-invariant functions in $\mathcal{D}(G)$, resp. $C^{\infty}(G), \mathcal{D}_{K}^{\prime}(G)$ the dual of $\mathcal{D}_{K}(G)$, identified with the space of left and right $\mathrm{K}$-invariant distributions in $\mathcal{D}^{\prime}(G)$, and similarly $\mathcal{D}_{W_{0}}(A)$, resp. $\mathcal{D}_{W_{0}}^{\prime}(A)$, the space of $W_{0}$-invariant elements of $\mathcal{D}(A)$, resp. $\mathcal{D}^{\prime}(A)$. Let

$$
F_{f}(a)=a^{\rho} \int_{N} f(a n) d n
$$

$f \in \mathcal{D}_{K}(G), a \in A$; then the map $f \mapsto F_{f}$ is an isomorphism of $\mathcal{D}_{K}(G)$ onto $\mathcal{D}_{W_{0}}(A)$ for the Schwartz topologies $([\mathbf{7}$, Cor. 7.9]). If $P \in \mathcal{Z}(\mathfrak{g})$, $F_{P f}=\gamma_{\mathfrak{a}}(P) F_{f}\left(\left[\mathbf{7}\right.\right.$, p. 307]). Transposing $F^{-1}$ we get an isomorphism $F^{t}$ of $\mathcal{D}_{K}^{\prime}(G)$ onto $\mathcal{D}_{W_{0}}^{\prime}(A)$, and

$$
F_{P T}^{t}=\gamma_{\mathfrak{a}}(P) F_{T}^{t}
$$

for all $P \in \mathcal{Z}(\mathfrak{g}), T \in \mathcal{D}_{K}^{\prime}(G)$.

Now let $P \in \mathcal{Z}\left(\mathcal{U}(\mathfrak{g})^{K}\right)$. If $\tau \in \hat{K}$, we can form the homomorphism $\gamma_{\mathfrak{a}} \otimes \chi_{\tau}: \mathcal{Z}\left(\mathcal{U}(\mathfrak{g})^{K}\right) \longrightarrow \mathcal{S}(\mathfrak{a})^{W_{0}}$.

Proposition 11.1. Let $G$ be a connected semisimple Lie group and $P \in$ $\mathcal{Z}\left(\mathcal{U}(\mathfrak{g})^{K}\right)$. If $P$ has a parametrix in $G$, then for all $\tau \in \hat{K},\left(\gamma_{\mathfrak{a}} \otimes \chi_{\tau}\right)(P) \neq 0$.

Proof. Suppose that $P$ has a parametrix $E$ and that exists $\tau \in \hat{K}$ such that $\left(\gamma_{\mathfrak{a}} \otimes \chi_{\tau}\right)(P)=0$. 
An easy computation shows that if $\Omega \in \mathcal{Z}(\mathfrak{k})$, then $\Omega E^{\tau}=\chi_{\tau}(\Omega) E^{\tau}$, so if $P^{\tau}=\left(I d \otimes \chi_{\tau}\right)(P) \in \mathcal{Z}(\mathfrak{g})$, then

$$
P E^{\tau}=P^{\tau} E^{\tau} \text {. }
$$

Taking $\tau$-components, $P E-\delta \in C^{\infty}(G)$ implies $P^{\tau} E^{\tau}-\delta^{\tau} \in C^{\infty}(G)$, and making everything $K$-bi-invariant we get $P^{\tau} E_{K}^{\tau}-\delta_{K}^{\tau} \in C_{K}^{\infty}(G)$.

Applying $F^{t}$ yields

$$
\gamma_{\mathfrak{a}}\left(P^{\tau}\right) F_{E_{K}^{\tau}}^{t}-F_{\delta_{K}^{\tau}}^{t} \in C_{W_{0}}^{\infty}(A)
$$

now $\gamma_{\mathfrak{a}}\left(P^{\tau}\right)=\left(\gamma_{\mathfrak{a}} \otimes \chi_{\tau}\right)(P)=0$ would imply $\delta_{K}^{\tau} \in C_{K}^{\infty}(G)$, which is absurd because it's easy to see that $\delta^{\tau}=d_{\tau} \Theta_{\tau} m_{K}$, where $m_{K}(f)=\int_{K} f$ is the distribution induced by the Haar measure of $K$.

\section{References}

[1] J. Arthur, Harmonic analysis of tempered distributions on semisimple Lie groups of real rank one, Thesis, Yale, 1970.

[2] A. Benabdallah, L'opérateur de Casimir de $S L(2, \mathbb{R})$, Ann. Scient. Éc. Norm. Sup., 17 (1984), 269-291.

[3] A. Benabdallah and F. Rouvière, Solvability of bi-invariant operators on a semisimple Lie group, C. R. Acad. Sc. Paris, 298(17), Serie I, (1984), 405-408.

[4] A. Cerezo and F. Rouvière, Solution élémentaire d'un opérateur invariant à gauche sur un groupe de Lie réel compact, Ann. Scient. Éc. Norm. Sup., 2 (1969), 561-581.

[5] _ Sur certains opérateurs différentiels invariants du groupe hyperbolique, Ann. Scient. Éc. Norm. Sup., 5 (1972), 581-597.

[6] Harish-Chandra, Harmonic analysis on real reductive groups III, Ann. of Math., 104 (1976), 117-201.

[7] S. Helgason, Groups and geometric analysis, Academic Press, Orlando, 1984.

[8] L. Hormander, The analysis of linear partial differential operators I, Springer-Verlag, Berlin, 1983.

[9] K. Johnson, Differential equations and an analog to Paley-Wiener theorem for linear semisimple Lie groups, Nagoya Math. Journal, 64 (1976), 17-29.

[10] A. Knapp, Representation theory of semisimple groups, Princeton University Press, 1986.

[11] F. Knop, Harish-Chandra homomorphisms for reductive group actions, Ann. of Math., 140 (1994), 253-288.

[12] J. Rauch and D. Wigner, Global solvability of the Casimir operator, Ann. of Math., 103(2) (1976), 229-236.

[13] F. Rouvière, Invariant differential equations on certain semisimple Lie groups, Transactions of the A.M.S., 243 (Sept. 1978), 97-115.

[14] N. Wallach, Harmonic analysis on homogeneous spaces, Marcel Dekker, New York, 1973.

[15] G. Warner, Harmonic analysis on semisimple Lie groups I, Springer-Verlag, Berlin, 1972 . 
[16] G. Zuckerman, Some characters identities for semisimple Lie groups, Thesis, Princeton, 1974.

Received April 20, 1999 and revised September 14, 1999. This work was supported by CONICET and FAMAF.

FAMAF - Universidad NACIONAL DE CóRdobA (5000) Córdoba

Argentina

E-mail address: ames@mate.uncor.edu 